JARosŁaW L. BoJARski (Warszawa)

\title{
THE RELAXATION OF THE SIGNORINI PROBLEM FOR POLYCONVEX FUNCTIONALS WITH LINEAR GROWTH AT INFINITY
}

Abstract. The aim of this paper is to study the unilateral contact condition (Signorini problem) for polyconvex functionals with linear growth at infinity. We find the lower semicontinuous relaxation of the original functional (defined over a subset of the space of bounded variations $B V(\Omega)$ ) and we prove the existence theorem. Moreover, we discuss the Winkler unilateral contact condition. As an application, we show a few examples of elastic-plastic potentials for finite displacements.

1. Introduction. Let $\Omega$ be a bounded, open, connected set of class $C^{1}$ in $\mathbb{R}^{3}$. The aim of this paper is to study the polyconvex functional

$$
\mathbf{u} \mapsto \int_{\Omega} j(x, \nabla \mathbf{u}, \operatorname{Adj} \nabla \mathbf{u}, \operatorname{det} \nabla \mathbf{u}) d x \in \mathbb{R} \cup\{\infty\}
$$

with unilateral contact conditions, where $j: \Omega \times\left(\mathbb{R}^{3 \times 3} \times \mathbb{R}^{3 \times 3} \times \mathbb{R}\right) \rightarrow$ $\mathbb{R} \cup\{\infty\}$ is a convex normal integrand, as considered in [3], [15] and in [12, Chapter 8, p. 232], and $\mathbf{u}$ is the displacement field. Adj $\nabla \mathbf{u}$ is the matrix of cofactors of $\nabla \mathbf{u}$ (cf. [3] and [15]). Note that $\nabla \mathbf{u}, \operatorname{Adj} \nabla \mathbf{u}$ and $\operatorname{det} \nabla \mathbf{u}$ govern the deformations of line, surface and volume elements, respectively. We are going to establish the lower semicontinuous (l.s.c.) relaxation of the functional (1.1), with unilateral contact conditions.

The motivation for this research is the necessity to study the polyconvex functionals encountered in deformational plasticity (for finite displacements). Polyconvex functionals have been investigated in [3], [15], [16], [17], [19] and [5] but without considering the unilateral contact conditions in the case of functionals with linear growth at infinity.

2000 Mathematics Subject Classification: 26B30, 46A11, 47H04, 49J45, 74B20, 74C15.

Key words and phrases: nonlinear elasticity, lower semicontinuous relaxation of a polyconvex functional, Signorini problem, Winkler's unilateral contact condition, variations. 
Here we establish the l.s.c. relaxation of the functional (1.1), defined on a subset of the space of bounded variations $B V(\Omega)$.

The existence theorem in nonlinear elasticity is given in [3] (see also [20]).

Dal Maso and Sbordone [9] prove the lower semicontinuity of a polyconvex functional with linear growth. However, the functional they consider is defined on the Sobolev space $W^{1, n}\left(\Omega, \mathbb{R}^{n}\right)$.

A geometric approach to the existence theorem for polyconvex functionals with linear growth at infinity is shown in [15]-[17]. In those papers it is proved that the minimum of a polyconvex functional belongs to the smallest sequentially closed set containing the graphs of certain $C^{1}$-mappings, given by transfinite induction (see [16]). In our paper, the solution is the limit of a simple sequence of $C^{1}$ functions in the weak* topology.

We find a new, explicit form of the relaxed functional $\widetilde{F}^{* *}$, unknown to the authors of [15].

Quasiconvex functionals defined on the $B V(\Omega)$ space have been investigated in [1], [2], [6], [14], but for normal integrands linear with respect to $\nabla \mathbf{u}$. Therefore those papers do not describe polyconvex problems, where the normal integrand depends non-linearly on $\operatorname{Adj} \nabla \mathbf{u}$ or det $\nabla \mathbf{u}$. In [7] quasiconvex functionals defined on $B V(\Omega)$ are considered, but with coercive conditions on the boundary and with the normal integrand satisfying the linear growth conditions.

Note that the unilateral contact conditions considered here are not coercive.

In this paper we extend the method of [5], where unilateral contact conditions are not considered. We take an alternative approach to that in [5]. We find the l.s.c. relaxation of the functional with linear growth at infinity and with unilateral contact conditions (the Signorini and Winkler problems). Moreover we prove the existence theorem.

In Section 3 we give examples of potentials which describe the deformational model of an elastic-plastic body under finite displacement. The functional $j_{4}$, defined in Example 3, has linear growth at infinity and depends nonlinearly on both $\nabla \mathbf{u}$ and $\operatorname{Adj} \nabla \mathbf{u}$.

2. Some basic definitions and theorems. Throughout this work $\Omega$ denotes a nonempty, bounded, open, connected subset of $\mathbb{R}^{3}$, with boundary of class $C^{1}$. $C\left(\bar{\Omega}, \mathbb{R}^{m}\right)$ denotes the space of $\mathbb{R}^{m}$-valued continuous functions on $\bar{\Omega}$, while $C_{0}\left(\Omega, \mathbb{R}^{m}\right)$, or simply $C_{0}$, stands for the space of continuous functions which are zero on $\partial \Omega$. The space $C_{c}\left(\Omega, \mathbb{R}^{m}\right)$, or simply $C_{c}$, is the space of $\mathbb{R}^{m}$-valued continuous functions with compact supports. The notation $\mathbb{M}_{b}\left(\Omega, \mathbb{R}^{m}\right)$, or $\mathbb{M}_{b}$, stands for the space of $\mathbb{R}^{m}$-valued, Radon bounded regular measures on $\Omega$, equipped with the norm $\|\cdot\|_{\mathbb{M}_{b}\left(\Omega, \mathbb{R}^{m}\right)}$. 
Moreover, we will use one of the duality pairs $\left(\mathbb{M}_{r}, C_{c}\right)$ or $\left(\mathbb{M}_{b}, C_{0}\right)$, where $\mathbb{M}_{r}$ is the space of regular measures. For $\mathbf{g}=\left(g_{1}, \ldots, g_{m}\right) \in C\left(\bar{\Omega}, \mathbb{R}^{m}\right)$ and $\boldsymbol{\mu}=\left(\mu_{1}, \ldots, \mu_{m}\right) \in \mathbb{M}_{b}\left(\Omega, \mathbb{R}^{m}\right)$, we write

$$
\int_{\Omega} \mathbf{g} \cdot \boldsymbol{\mu} \equiv \sum_{i=1}^{m} \int_{\Omega} g_{i} \mu_{i} .
$$

Finally, $\mathcal{L}^{0}\left(\Omega, \mathbb{R}^{m}\right)_{\mu}$ stands for the set of $\mu$-measurable functions from $\Omega$ into $\mathbb{R}^{m}$.

The scalar product of $\mathbf{z}, \mathbf{z}^{*} \in \mathbb{R}^{m}$ is denoted by $\mathbf{z} \cdot \mathbf{z}^{*}=\sum_{i} z^{i} z_{i}^{*}$ and the scalar product of $\mathbf{w}, \mathbf{w}^{*} \in \mathbb{R}^{m \times m} \equiv \mathbb{E}^{m}$ by $\mathbf{w}: \mathbf{w}^{*}=\sum_{i j} w^{i j} w_{i j}^{*}$, where $\mathbb{E}^{m}$ is the space of real $m \times m$ matrices.

Let $X$ be a vector space, and $\tau \subset 2^{X}$ a linear topology on $X$ (or a norm on $X)$. Then $[X, \tau]$ denotes the topological space and $[X, \tau]^{*}$ its dual space. If $F: X \rightarrow \mathbb{R} \cup\{\infty\}$ is a function defined on $X$, then $F^{*}$ denotes its polar function (cf. [12]). For an arbitrary set $C$ in $X, I_{C}(\cdot)$ stands for its indicator function $\left(I_{C}(x)=0\right.$ if $x \in C$ and $I_{C}(x)=+\infty$ if $\left.x \notin C\right)$.

We also need the following notations. Let $V$ be a metric space. Then $B_{V}(\Xi, r)$ is the closed ball in $V$ with center $\Xi$ and radius $r$. The notation $\mathrm{cl}_{V}(Z)$ stands for the closure of the set $Z \subset V$ in the topology of $V$, while, analogously, $\mathrm{cl}_{\|\cdot\|}(Z)$ is the closure of $Z$ in the norm $\|\cdot\|$. In our paper we take $\left\|\left[e_{i}\right]\right\|_{\mathbb{R}^{m}} \equiv \sum_{i=1}^{m}\left|e_{i}\right|$ for $\left[e_{i}\right] \in \mathbb{R}^{m}$. The tensor product is denoted by $\otimes$.

We define the following Banach spaces (see [18], [23] and [24]):

$$
\begin{gathered}
B V\left(\Omega, \mathbb{R}^{3}\right) \equiv\left\{\mathbf{u} \in L^{1}\left(\Omega, \mathbb{R}^{3}\right) \mid \nabla \mathbf{u} \in \mathbb{M}_{b}\left(\Omega, \mathbb{E}^{3}\right)\right\} \\
B D(\Omega) \equiv\left\{\mathbf{u} \in L^{1}\left(\Omega, \mathbb{R}^{3}\right) \mid \varepsilon_{i j}(\mathbf{u}) \equiv \frac{1}{2}\left(\frac{\partial u_{i}}{\partial x_{j}}+\frac{\partial u_{j}}{\partial x_{i}}\right) \in \mathbb{M}_{b}(\Omega), i, j=1,2,3\right\}
\end{gathered}
$$

with the natural norms

$$
\|\mathbf{u}\|_{B V}=\|\mathbf{u}\|_{L^{1}}+\sum_{i, j}^{3}\left\|\frac{\partial u_{i}}{\partial x_{j}}\right\|_{\mathbb{M}_{b}}, \quad\|\mathbf{u}\|_{B D}=\|\mathbf{u}\|_{L^{1}}+\sum_{i, j}^{3}\left\|\varepsilon_{i j}(\mathbf{u})\right\|_{\mathbb{M}_{b}} .
$$

Proposition 1 (see [18], [23]). Let $B V(\Omega)$ and $L^{1}\left(\partial \Omega, \mathbb{R}^{3}\right)$ be endowed with their respective norm topologies. Then there exists a continuous surjective linear trace operator $\gamma$ from $B V(\Omega)$ onto $L^{1}\left(\partial \Omega, \mathbb{R}^{3}\right)$ such that $\gamma(\mathbf{u})=$ $\mathbf{u}_{\mid \partial \Omega}$ for all $\mathbf{u} \in B V(\Omega) \cap C\left(\bar{\Omega}, \mathbb{R}^{3}\right)$.

The trace $\gamma: B V(\Omega) \rightarrow L^{1}\left(\partial \Omega, \mathbb{R}^{3}\right)$ is a surjection, since the trace from $W^{1,1}\left(\Omega, \mathbb{R}^{3}\right)$ to $L^{1}\left(\partial \Omega, \mathbb{R}^{3}\right)$ is a surjection.

In this paper, $\Omega$ and $\Omega_{1}$ are bounded open connected sets of class $C^{1}$ in $\mathbb{R}^{3}$ such that $\Omega \subset \subset \Omega_{1}$. The Lebesgue and Hausdorff measures on $\Omega$ and $\partial \Omega$ are denoted by $d x$ and $d s$, respectively. The Hausdorff measure $d s$ multiplied by a real constant equals the 2 -dimensional Lebesgue measure (on the hyperplane $\mathbb{R}^{2}$ ). 
Assumption 1 (cf. [4]). $\mathcal{K}: \bar{\Omega} \rightarrow 2^{\mathbb{E}^{3} \times \mathbb{E}^{3} \times \mathbb{R}}$ is a multifunction with convex closed values. Moreover, there exists $\widetilde{\mathbf{z}} \in C^{1}\left(\bar{\Omega}, \mathbb{E}^{3} \times \mathbb{E}^{3} \times \mathbb{R}\right)$ such that $\widetilde{\mathbf{z}}(x) \in \mathcal{K}(x)$ for every $x \in \bar{\Omega}$, and the following conditions hold:

(i) if $\mathbf{z} \in C^{1}\left(\bar{\Omega}, \mathbb{E}^{3} \times \mathbb{E}^{3} \times \mathbb{R}\right)$ and $\mathbf{z}(x) \in \mathcal{K}(x)$ for $d x$-almost every (dx-a.e.) $x \in \Omega$, then $\mathbf{z}(y) \in \mathcal{K}(y)$ for every $y \in \bar{\Omega}$;

(ii) for every $y \in \bar{\Omega}$ and every $\mathbf{w} \in \mathcal{K}(y)$ there exists $\mathbf{z} \in C^{1}\left(\bar{\Omega}, \mathbb{E}^{3} \times\right.$ $\left.\mathbb{E}^{3} \times \mathbb{R}\right)$ such that $\mathbf{z}(y)=\mathbf{w}$ and $\mathbf{z}(x) \in \mathcal{K}(x)$ for every $x \in \bar{\Omega}$.

Conditions (i) and (ii) are equivalent to the condition that for every $y \in \bar{\Omega}$,

$$
\begin{aligned}
\mathcal{K}(y)=\left\{\mathbf{z}(y) \mid \mathbf{z} \in C^{1}\left(\bar{\Omega}, \mathbb{E}^{3} \times \mathbb{E}^{3} \times \mathbb{R}\right),\right. \\
\quad \mathbf{z}(x) \in \mathcal{K}(x) \text { for } d x \text {-a.e. } x \in \Omega\} .
\end{aligned}
$$

Definition 1 (cf. [12, Chapter 8, p. 232]). Let $j^{*}: \Omega \times\left(\mathbb{E}^{3} \times \mathbb{E}^{3} \times \mathbb{R}\right) \rightarrow$ $\mathbb{R} \cup\{\infty\}$ be a convex, nonnegative, normal integrand, i.e.:

(a) $\mathbb{E}^{3} \times \mathbb{E}^{3} \times \mathbb{R} \ni \mathbf{w}^{*} \mapsto j^{*}\left(x, \mathbf{w}^{*}\right)$ is convex and l.s.c. for $d x$-a.e. $x \in \Omega$;

(b) there exists a Borel function $\tilde{j}^{*}: \Omega \times\left(\mathbb{E}^{3} \times \mathbb{E}^{3} \times \mathbb{R}\right) \rightarrow \mathbb{R} \cup\{\infty\}$ such that $j^{*}(x, \cdot)=j^{*}(x, \cdot)$ for $d x$-a.e. $x \in \Omega$.

We also assume that

$$
\left\{\mathbf{w}^{*} \in \mathbb{E}^{3} \times \mathbb{E}^{3} \times \mathbb{R} \mid j^{*}\left(x, \mathbf{w}^{*}\right)<\infty\right\}=\mathcal{K}(x)
$$

for $d x$-a.e. $x \in \Omega$.

Assumption 2. There exist constants $k, r_{1}>0$ such that

$$
j^{*}\left(x, \mathbf{w}^{*}\right) \leq k, \quad \forall \mathbf{w}^{*} \in B_{\mathbb{E}^{3} \times \mathbb{E}^{3} \times \mathbb{R}}\left(0, r_{1}\right), \text { for } d x \text {-a.e. } x \in \Omega,
$$

where $B_{\mathbb{E}^{3} \times \mathbb{E}^{3} \times \mathbb{R}}\left(0, r_{1}\right)$ is the closed ball in $\mathbb{E}^{3} \times \mathbb{E}^{3} \times \mathbb{R}$ with center 0 and radius $r_{1}$ (in the finite-dimensional space all norms are equivalent). Moreover, we assume that for every $\widehat{r}>0$ there exists $c_{\widehat{r}}<\infty$ such that

$$
\begin{aligned}
& \sup \left\{\int_{\Omega} j^{*}\left(x, \mathbf{w}^{*}\right) d x \mid \mathbf{w}^{*} \in L^{\infty}\left(\Omega, \mathbb{E}^{3} \times \mathbb{E}^{3} \times \mathbb{R}\right),\right. \\
& \left.\left\|\mathbf{w}^{*}\right\|_{L^{\infty}\left(\Omega, \mathbb{E}^{3} \times \mathbb{E}^{3} \times \mathbb{R}\right)}<\widehat{r} \text { and } \mathbf{w}^{*}(x) \in \mathcal{K}(x) \text { for } d x \text {-a.e. } x \in \Omega\right\}<c_{\widehat{r}} .
\end{aligned}
$$

By $(2.2)-(2.4)$,

$$
B_{\mathbb{E}^{3} \times \mathbb{E}^{3} \times \mathbb{R}}\left(0, r_{1}\right) \subset \mathcal{K}(x), \quad \forall x \in \bar{\Omega} .
$$

The Legendre-Fenchel transform of $j^{*}$ is given by

$$
\begin{array}{r}
j^{* *}(x, \mathbf{z}, \mathbf{p}, t) \equiv \sup \left\{\mathbf{z}: \mathbf{z}^{*}+\mathbf{p}: \mathbf{p}^{*}+t t^{*}-j^{*}\left(x, \mathbf{z}^{*}, \mathbf{p}^{*}, t^{*}\right) \mid\right. \\
\left.\mathbf{z}^{*} \in \mathbb{E}^{3}, \mathbf{p}^{*} \in \mathbb{E}^{3}, t^{*} \in \mathbb{R}\right\}
\end{array}
$$

for $d x$-a.e. $x \in \Omega$ and every $(\mathbf{z}, \mathbf{p}, t) \in \mathbb{E}^{3} \times \mathbb{E}^{3} \times \mathbb{R}$. Moreover, the functional $j: \Omega \times\left(\mathbb{E}^{3} \times \mathbb{E}^{3} \times \mathbb{R}\right) \rightarrow \mathbb{R} \cup\{\infty\}$ is defined by

$$
j(x, \mathbf{z}, \mathbf{p}, t) \equiv j^{* *}(x, \mathbf{z}, \mathbf{p}, t) \quad \text { for } d x \text {-a.e. } x \in \Omega, \forall(\mathbf{z}, \mathbf{p}, t) \in \mathbb{E}^{3} \times \mathbb{E}^{3} \times \mathbb{R} \text {. }
$$


In view of $(2.4)$,

$$
j(x, \mathbf{z}, \mathbf{p}, t) \geq c r_{1}\left(\|\mathbf{z}\|_{\mathbb{E}^{3}}+\|\mathbf{p}\|_{\mathbb{E}^{3}}+|t|\right)-k
$$

for every $(\mathbf{z}, \mathbf{p}, t) \in \mathbb{E}^{3} \times \mathbb{E}^{3} \times \mathbb{R}$ and $d x$-a.e. $x \in \Omega$. Here $c$ is a positive constant (cf. the definition of $\|\cdot\|_{\mathbb{R}^{3 \times 3}}=\|\cdot\|_{\mathbb{E}^{3}}$ ). Since $j^{*}$ is a nonnegative function, we observe that

$$
0 \geq j(x, \mathbf{0}, \mathbf{0}, 0) \quad \text { for } d x \text {-a.e. } x \in \Omega \text {. }
$$

Let $j_{\infty}: \bar{\Omega} \times \mathbb{E}^{3} \times \mathbb{E}^{3} \times \mathbb{R} \rightarrow \mathbb{R} \cup\{\infty\}$ be defined by

$$
\begin{aligned}
j_{\infty}(x, \mathbf{z}, \mathbf{p}, t) \equiv \sup \left\{\mathbf{z}: \mathbf{z}^{*}+\mathbf{p}: \mathbf{p}^{*}+t t^{*}-I_{\mathcal{K}(x)}\left(\mathbf{z}^{*}, \mathbf{p}^{*}, t^{*}\right) \mid\right. \\
\left.\quad \mathbf{z}^{*} \in \mathbb{E}^{3}, \mathbf{p}^{*} \in \mathbb{E}^{3}, t^{*} \in \mathbb{R}\right\}
\end{aligned}
$$

for $(x, \mathbf{z}, \mathbf{p}, t) \in \bar{\Omega} \times \mathbb{E}^{3} \times \mathbb{E}^{3} \times \mathbb{R}$. Then

$$
j_{\infty}(x, \mathbf{z}, \mathbf{p}, t) \geq c r_{1}\left(\|\mathbf{z}\|_{\mathbb{E}^{3}}+\|\mathbf{p}\|_{\mathbb{E}^{3}}+|t|\right), \quad \forall(x, \mathbf{z}, \mathbf{p}, t) \in \bar{\Omega} \times \mathbb{E}^{3} \times \mathbb{E}^{3} \times \mathbb{R},
$$

where $c$ is a positive constant (cf. the definition of $\|\cdot\|_{\mathbb{E}^{3}}$ ).

Definition 2. For every $\mathbf{u} \in B V\left(\Omega, \mathbb{R}^{3}\right), \widetilde{\boldsymbol{\mu}} \in \mathbb{M}_{b}\left(\Omega, \mathbb{E}^{3}\right)$ and $\mu \in$ $\mathbb{M}_{b}(\Omega, \mathbb{R})$, we define

$$
\|(\mathbf{u}, \widetilde{\boldsymbol{\mu}}, \mu)\|_{P^{3} B V}=\|\mathbf{u}\|_{B V}+\|\widetilde{\boldsymbol{\mu}}\|_{\mathbb{M}_{b}\left(\Omega, \mathbb{E}^{3}\right)}+\|\mu\|_{\mathbb{M}_{b}(\Omega, \mathbb{R})},
$$

where $\|\widetilde{\boldsymbol{\mu}}\|_{\mathbb{M}_{b}\left(\Omega, \mathbb{E}^{3}\right)}=\sum_{i j}^{3}\left\|\widetilde{\mu}_{i j}\right\|_{\mathbb{M}_{b}(\Omega, \mathbb{R})}$ and $\widetilde{\mu}_{i j}$ are the components of $\tilde{\boldsymbol{\mu}}$.

Definition 3. For every $\boldsymbol{\omega}^{0} \in C\left(\bar{\Omega}, \mathbb{E}^{3}\right), \boldsymbol{\omega}^{1} \in C_{0}\left(\Omega, \mathbb{E}^{3}\right), \omega^{2} \in C_{0}(\Omega, \mathbb{R})$, $\mathbf{u} \in B V\left(\Omega, \mathbb{R}^{3}\right), \widetilde{\boldsymbol{\mu}} \in \mathbb{M}_{b}\left(\Omega, \mathbb{E}^{3}\right)$ and $\mu \in \mathbb{M}_{b}(\Omega, \mathbb{R})$ we define a bilinear functional by

$$
\begin{aligned}
\left\langle(\gamma(\mathbf{u}), \nabla \mathbf{u}, \widetilde{\boldsymbol{\mu}}, \mu),\left(\boldsymbol{\omega}^{0}, \boldsymbol{\omega}^{1}, \omega^{2}\right)\right\rangle_{P^{3} B V}= & \int_{\Omega} \boldsymbol{\omega}^{0}: \nabla \mathbf{u}+\int_{\Omega} \boldsymbol{\omega}^{1}: \widetilde{\boldsymbol{\mu}} \\
& +\int_{\Omega} \omega^{2} \mu-\int_{\partial \Omega} \boldsymbol{\beta}\left(\boldsymbol{\omega}^{0}\right) \cdot \gamma(\mathbf{u}) d s
\end{aligned}
$$

where $\boldsymbol{\beta}\left(\boldsymbol{\omega}^{0}\right)=\boldsymbol{\omega}^{0} \cdot \boldsymbol{\nu}$ on $\partial \Omega$ and $\boldsymbol{\nu}$ is the exterior unit normal to $\partial \Omega$.

Let $\Gamma_{S}, \Gamma_{W}, \Gamma_{0}$ and $\Gamma_{1}$ (with $\Gamma_{1}=\bar{\Gamma}_{1}$ and $\Gamma_{S} \cup \Gamma_{W}=\overline{\Gamma_{S} \cup \Gamma_{W}}-\bar{\Gamma}_{1}$ ) be Borel subsets of $\partial \Omega$ such that $\Gamma_{1} \cap \Gamma_{0}=\emptyset=\Gamma_{0} \cap \Gamma_{S}, \Gamma_{1} \cap \Gamma_{S}=\emptyset=\Gamma_{1} \cap \Gamma_{W}$, $\Gamma_{0} \cap \Gamma_{W}=\emptyset=\Gamma_{W} \cap \Gamma_{S}$ and $\Gamma_{S} \cup \Gamma_{W} \cup \Gamma_{1} \cup \Gamma_{0}=\partial \Omega$. Moreover, let $d s\left(\operatorname{int} \Gamma_{0}\right)=d s\left(\bar{\Gamma}_{0}\right), d s\left(\operatorname{int} \Gamma_{S}\right)=d s\left(\bar{\Gamma}_{S}\right)$ and $d s\left(\operatorname{int} \Gamma_{W}\right)=d s\left(\bar{\Gamma}_{W}\right)$.

On the boundary $\Gamma_{S}$ we have the Signorini contact condition $\boldsymbol{\nu}(x)$. $\gamma(\mathbf{u})(x) \leq 0$ for $d s$-a.e. $x \in \Gamma_{S}$ (see [11]). This means that we have a potential function $h_{S}: \Gamma_{S} \times \mathbb{E}^{3} \rightarrow \mathbb{R} \cup\{\infty\}$ defined by

$$
h_{S}(x, \mathbf{y})= \begin{cases}\infty & \text { if } \sum_{i=1}^{3} y_{i i}=\operatorname{tr} \mathbf{y}<0 \\ 0 & \text { otherwise }\end{cases}
$$

for $x \in \Gamma_{S}$, where $\mathbf{y}=-\boldsymbol{\nu} \otimes \gamma(\mathbf{u})$ (cf. [4]). In (2.10), $h_{S}$ depends only on $\mathbf{y}$. 
On the boundary $\Gamma_{W}$ we consider the Winkler unilateral contact condition, given by the potential $h_{W}: \Gamma_{W} \times \mathbb{E}^{3} \rightarrow \mathbb{R} \cup\{\infty\}$, defined by

$$
h_{W}(x, \mathbf{y})= \begin{cases}\frac{1}{2} \alpha_{0}(x)(\operatorname{tr} \mathbf{y})^{2} & \text { if } \operatorname{tr} \mathbf{y}<0, \\ 0 & \text { otherwise }\end{cases}
$$

for every $x \in \Gamma_{W}$, where $\mathbf{y}=-\boldsymbol{\nu} \otimes \boldsymbol{\gamma}(\mathbf{u}), \alpha_{0} \in L^{\infty}\left(\Gamma_{W}, \mathbb{R}\right)$ and $\alpha_{0}(x)>0$ for $d s$-a.e. $x \in \Gamma_{W}$ (see [21]).

In what follows, we consider a functional $F: C^{1}\left(\bar{\Omega}, \mathbb{R}^{3}\right) \rightarrow \mathbb{R} \cup\{\infty\}$ given by

$$
\begin{aligned}
F(\mathbf{u})= & -\int_{\Gamma_{1}} \mathbf{g} \cdot \mathbf{u} d s+\int_{\Gamma_{0}} I_{\{\boldsymbol{\nu} \otimes \tilde{\mathbf{u}}=\mathbf{0}\}}(-\boldsymbol{\nu} \otimes \mathbf{u}) d s \\
& +\int_{\Gamma_{S}} h_{S}(x,-\boldsymbol{\nu} \otimes \mathbf{u}) d s+\int_{\Gamma_{W}} h_{W}(x,-\boldsymbol{\nu} \otimes \mathbf{u}) d s \\
& +\int_{\Omega} j(x, \nabla \mathbf{u}, \operatorname{Adj}(\nabla \mathbf{u}), \operatorname{det}(\nabla \mathbf{u})) d x,
\end{aligned}
$$

where $\mathbf{w} \mapsto I_{\{\boldsymbol{\nu} \otimes \widetilde{\mathbf{u}}=\mathbf{0}\}}(\mathbf{w})$ is the indicator function of the set $\left\{\boldsymbol{\nu} \otimes \widetilde{\mathbf{u}} \in \boldsymbol{\nu} \otimes \mathbb{R}^{3} \mid\right.$ $\boldsymbol{\nu} \otimes \widetilde{\mathbf{u}}=\mathbf{0}\}$.

Assumption 3. There exists $\boldsymbol{\sigma}_{0} \in C\left(\bar{\Omega}, \mathbb{E}^{3}\right)$ such that $\boldsymbol{\sigma}_{0} \cdot \boldsymbol{\nu}=\mathrm{g}$ on $\Gamma_{1}$, $\boldsymbol{\sigma}_{0}=\mathbf{0}$ on $\Gamma_{W} \cup \Gamma_{S}$ and $\left(\boldsymbol{\sigma}_{0}(x), \mathbf{0}, 0\right) \in \mathcal{K}(x)$ for every $x \in \bar{\Omega}$.

For an elastic-plastic material, the functional $F$ describes the total energy of a body occupying the given subset $\Omega$ of $\mathbb{R}^{3}$, and subjected to the boundary force $\mathbf{g}$ on $\Gamma_{1}$. The body is clamped on $\Gamma_{0}$, meaning that the Dirichlet condition $\mathbf{u}=\mathbf{0}$ holds on $\Gamma_{0}$. Moreover, the Signorini contact condition on $\Gamma_{S}$ and the Winkler contact condition on $\Gamma_{W}$ are assumed.

The extension $\widetilde{F}$ of $F$ to $C^{1}\left(\bar{\Omega}, \mathbb{R}^{3}\right) \times L^{1}\left(\Omega, \mathbb{E}^{3}\right) \times L^{1}\left(\Omega, \mathbb{E}^{3}\right) \times L^{1}(\Omega, \mathbb{R})$ is given by

$$
\begin{aligned}
& \widetilde{F}\left(\mathbf{u}_{\mid \partial \Omega}, \mathbf{z}, \mathbf{p}, t\right)=-\int_{\Gamma_{1}} \mathbf{g} \cdot \mathbf{u} d s+\int_{\Gamma_{0}} I_{\{\boldsymbol{\nu} \otimes \widetilde{\mathbf{u}}=\mathbf{0}\}}(-\boldsymbol{\nu} \otimes \mathbf{u}) d s \\
& \quad+\int_{\Gamma_{S}} h_{S}(x,-\boldsymbol{\nu} \otimes \mathbf{u}) d s+\int_{\Gamma_{W}} h_{W}(x,-\boldsymbol{\nu} \otimes \mathbf{u}) d s+\int_{\Omega} j(x, \mathbf{z}, \mathbf{p}, t) d x .
\end{aligned}
$$

A bilinear form between $C^{1}\left(\bar{\Omega}, \mathbb{R}^{3}\right) \times L^{1}\left(\Omega, \mathbb{E}^{3}\right) \times L^{1}\left(\Omega, \mathbb{E}^{3}\right) \times L^{1}(\Omega, \mathbb{R})$ and $C\left(\bar{\Omega}, \mathbb{E}^{3}\right) \times C_{0}\left(\Omega, \mathbb{E}^{3}\right) \times C_{0}(\Omega, \mathbb{R})$ is

$$
\begin{aligned}
\left\langle\left(\mathbf{u}_{\mid \partial \Omega}, \mathbf{z}, \mathbf{p}, t\right),\left(\boldsymbol{\omega}^{0}, \boldsymbol{\omega}^{1}, \omega^{2}\right)\right\rangle= & \int_{\Omega} \boldsymbol{\omega}^{0}: \mathbf{z} d x+\int_{\Omega} \boldsymbol{\omega}^{1}: \mathbf{p} d x \\
& +\int_{\Omega} \omega^{2} t d x-\int_{\partial \Omega} \boldsymbol{\omega}^{0}:\left(\boldsymbol{\nu} \otimes \mathbf{u}_{\mid \partial \Omega}\right) d s,
\end{aligned}
$$

where $\mathbf{u} \in C^{1}\left(\bar{\Omega}, \mathbb{R}^{3}\right), \mathbf{z} \in L^{1}\left(\Omega, \mathbb{E}^{3}\right), \mathbf{p} \in L^{1}\left(\Omega, \mathbb{E}^{3}\right), t \in L^{1}(\Omega, \mathbb{R}), \boldsymbol{\omega}^{0} \in$ $C\left(\bar{\Omega}, \mathbb{E}^{3}\right), \boldsymbol{\omega}^{1} \in C_{0}\left(\Omega, \mathbb{E}^{3}\right)$ and $\omega^{2} \in C_{0}(\Omega, \mathbb{R})$. 
We mention that the dual functional $\widetilde{F}^{*}: C\left(\bar{\Omega}, \mathbb{E}^{3}\right) \times C_{0}\left(\Omega, \mathbb{E}^{3}\right) \times C_{0}(\Omega, \mathbb{R})$ $\rightarrow \mathbb{R} \cup\{\infty\}$ is defined by

$$
\begin{array}{r}
\widetilde{F}^{*}\left(\boldsymbol{\omega}^{0}, \boldsymbol{\omega}^{1}, \omega^{2}\right)=\sup \left\{\left\langle\left(\mathbf{u}_{\mid \partial \Omega}, \mathbf{z}, \mathbf{p}, t\right),\left(\boldsymbol{\omega}^{0}, \boldsymbol{\omega}^{1}, \omega^{2}\right)\right\rangle-\widetilde{F}\left(\mathbf{u}_{\mid \partial \Omega}, \mathbf{z}, \mathbf{p}, t\right) \mid\right. \\
\left.(\mathbf{u}, \mathbf{z}, \mathbf{p}, t) \in C^{1}\left(\bar{\Omega}, \mathbb{R}^{3}\right) \times L^{1}\left(\Omega, \mathbb{E}^{3}\right) \times L^{1}\left(\Omega, \mathbb{E}^{3}\right) \times L^{1}(\Omega, \mathbb{R})\right\}
\end{array}
$$

for $\left(\boldsymbol{\omega}^{0}, \boldsymbol{\omega}^{1}, \omega^{2}\right) \in C\left(\bar{\Omega}, \mathbb{E}^{3}\right) \times C_{0}\left(\Omega, \mathbb{E}^{3}\right) \times C_{0}(\Omega, \mathbb{R})$.

The bidual functional $\widetilde{F}^{* *}: B V(\Omega) \times \mathbb{M}_{b}\left(\Omega, \mathbb{E}^{3}\right) \times \mathbb{M}_{b}(\Omega, \mathbb{R}) \rightarrow \mathbb{R} \cup\{\infty\}$ is given by

$$
\begin{aligned}
& \widetilde{F}^{* *}(\boldsymbol{\gamma}(\mathbf{u}), \nabla \mathbf{u}, \widetilde{\boldsymbol{\mu}}, \mu)=\sup \left\{\left\langle(\gamma(\mathbf{u}), \nabla \mathbf{u}, \widetilde{\boldsymbol{\mu}}, \mu),\left(\boldsymbol{\omega}^{0}, \boldsymbol{\omega}^{1}, \omega^{2}\right)\right\rangle_{P^{3} B V}\right. \\
& \left.-\widetilde{F}^{*}\left(\boldsymbol{\omega}^{0}, \boldsymbol{\omega}^{1}, \omega^{2}\right) \mid \boldsymbol{\omega}^{0} \in C\left(\bar{\Omega}, \mathbb{E}^{3}\right), \boldsymbol{\omega}^{1} \in C_{0}\left(\Omega, \mathbb{E}^{3}\right), \omega^{2} \in C_{0}(\Omega, \mathbb{R})\right\}
\end{aligned}
$$

for $(\mathbf{u}, \widetilde{\boldsymbol{\mu}}, \mu) \in B V(\Omega) \times \mathbb{M}_{b}\left(\Omega, \mathbb{E}^{3}\right) \times \mathbb{M}_{b}(\Omega, \mathbb{R})$.

Definition 4. For $q>0$ we define a subset $P_{q}^{3} B V(\Omega)$ of $B V\left(\Omega, \mathbb{R}^{3}\right) \times$ $\mathbb{M}_{b}\left(\Omega, \mathbb{E}^{3}\right) \times \mathbb{M}_{b}(\Omega, \mathbb{R})$ to consist of $(\mathbf{u}, \tilde{\boldsymbol{\mu}}, \mu)$ for which $\|(\mathbf{u}, \widetilde{\boldsymbol{\mu}}, \mu)\|_{P^{3} B V} \leq q$ and for which there exists a sequence $\left\{\mathbf{u}_{n}\right\}_{n \in \mathbb{N}} \subset C^{1}\left(\bar{\Omega}, \mathbb{R}^{3}\right)$ such that

$$
\left\|\left(\mathbf{u}_{n}, \operatorname{Adj} \nabla \mathbf{u}_{n}, \operatorname{det} \nabla \mathbf{u}_{n}\right)\right\|_{P^{3} B V} \leq q, \quad \forall n \in \mathbb{N},
$$

and moreover

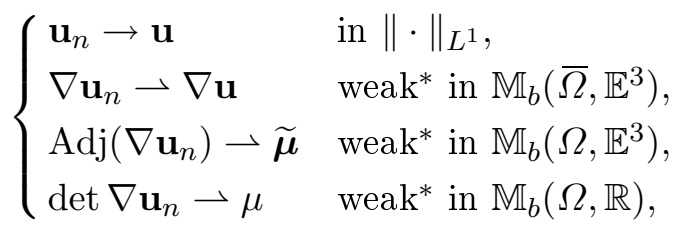

where $\mathbb{M}_{b}\left(\bar{\Omega}, \mathbb{E}^{3}\right) \equiv C\left(\bar{\Omega}, \mathbb{E}^{3}\right)^{*}$ (cf. [10, Theorem VI.7.2]).

We say that $\left\{\mathbf{u}_{n}\right\}_{n \in \mathbb{N}} \subset B V\left(\Omega, \mathbb{R}^{3}\right)$ converges to $\mathbf{u} \in B V\left(\Omega, \mathbb{R}^{3}\right)$ in the topology (2.18) if

$$
\begin{cases}\mathbf{u}_{n} \rightarrow \mathbf{u} & \text { in }\|\cdot\|_{L^{1}}, \\ \nabla \mathbf{u}_{n} \rightarrow \nabla \mathbf{u} & \text { weak }^{*} \text { in } \mathbb{M}_{b}\left(\Omega, \mathbb{E}^{3}\right) .\end{cases}
$$

Furthermore, a sequence $\left\{\left(\mathbf{u}_{n}, \tilde{\boldsymbol{\mu}}_{n}, \mu_{n}\right)\right\}_{n \in \mathbb{N}} \subset P_{q}^{3} B V(\Omega)$ is said to converge to $(\mathbf{u}, \tilde{\boldsymbol{\mu}}, \mu) \in P_{q}^{3} B V(\Omega)$ in the topology (2.18)-(2.19) if

$$
\begin{cases}\widetilde{\boldsymbol{\mu}}_{n} \rightarrow \widetilde{\boldsymbol{\mu}} & \text { weak }^{*} \text { in } \mathbb{M}_{b}\left(\Omega, \mathbb{E}^{3}\right), \\ \mu_{n} \rightarrow \mu & \text { weak* in } \mathbb{M}_{b}(\Omega, \mathbb{R})\end{cases}
$$

and $\left\{\mathbf{u}_{n}\right\}_{n \in \mathbb{N}}$ converges to $\mathbf{u}$ in the topology (2.18).

DEFINITION 5. Let

$$
A(x)=\left\{\boldsymbol{\omega}^{0} \in \mathbb{E}^{3} \mid \sum_{i, j=1}^{3}\left(\boldsymbol{\omega}^{0}\right)_{i j}(\boldsymbol{\nu} \otimes \mathbf{u})_{i j} \geq 0\right.
$$

for all $\mathbf{u}$ such that $\boldsymbol{\nu} \cdot \mathbf{u} \leq 0\}$

for $d s$-a.e. $x \in \Gamma_{S} \cup \Gamma_{W}$ (cf. [4, Section 4.6]). 
REMARK 1. Since

$$
\sum_{i, j=1}^{3} \omega_{i j}(\boldsymbol{\nu} \otimes \mathbf{u})_{i j}=\frac{1}{3} \operatorname{tr} \boldsymbol{\omega} \operatorname{tr}(\boldsymbol{\nu} \otimes \mathbf{u})+\sum_{i, j=1}^{3} \omega_{i j}^{D}(\boldsymbol{\nu} \otimes \mathbf{u})_{i j}^{D}
$$

where the deviator part of $\boldsymbol{\omega}$ is given by $\boldsymbol{\omega}^{D}=\boldsymbol{\omega}-\frac{1}{3}(\operatorname{tr} \boldsymbol{\omega}) \boldsymbol{\delta}$ and $\boldsymbol{\delta}$ is the Kronecker delta, it follows that

$$
A_{L} \equiv\left\{\boldsymbol{\omega}^{0} \in \mathbb{E}^{3} \mid \operatorname{tr} \boldsymbol{\omega}^{0} \leq 0,\left(\boldsymbol{\omega}^{0}\right)^{D}=\mathbf{0}\right\} \subset A(x)
$$

for $d s$-a.e. $x \in \Gamma_{S} \cup \Gamma_{W}$. The functional $h_{S}^{*}: \Gamma_{S} \times \mathbb{E}^{3} \rightarrow \mathbb{R} \cup\{\infty\}$ is given by

$$
h_{S}^{*}\left(x, \boldsymbol{\omega}^{0}\right)=\sup _{\mathbf{y}}\left\{\mathbf{y}: \boldsymbol{\omega}^{0}-h_{S}(x, \mathbf{y})\right\}= \begin{cases}0 & \text { if } \boldsymbol{\omega}^{0} \in A(x), \\ \infty & \text { otherwise }\end{cases}
$$

for every $\left(x, \boldsymbol{\omega}^{0}\right) \in \Gamma_{S} \times \mathbb{E}^{3}$. Similarly, the functional $h_{W}^{*}: \Gamma_{W} \times \mathbb{E}^{3} \rightarrow \mathbb{R} \cup\{\infty\}$ is given by

$$
\begin{aligned}
h_{W}^{*}\left(x, \boldsymbol{\omega}^{0}\right) & =\sup _{\mathbf{y}}\left\{\mathbf{y}: \boldsymbol{\omega}^{0}-h_{W}(x, \mathbf{y})\right\} \\
& = \begin{cases}\frac{1}{2 \alpha_{0}(x)}\left(\frac{1}{3} \operatorname{tr} \boldsymbol{\omega}^{0}\right)^{2} & \text { if } \boldsymbol{\omega}^{0} \in A(x), \\
\infty & \text { otherwise },\end{cases}
\end{aligned}
$$

for every $\left(x, \boldsymbol{\omega}^{0}\right) \in \Gamma_{W} \times \mathbb{E}^{3}$, where $A(x)$ is defined by (2.20).

Theorem 2. The functional $\widetilde{F}^{* *}$ defined by (2.16) satisfies

$$
\begin{aligned}
& \widetilde{F}^{* *}(\gamma(\mathbf{u}), \nabla \mathbf{u}, \widetilde{\boldsymbol{\mu}}, \mu)=-\int_{\Gamma_{1}} \mathbf{g} \cdot \boldsymbol{\gamma}(\mathbf{u}) d s \\
&+ \int_{\Gamma_{0}} j_{\infty}(x,-\boldsymbol{\nu} \otimes \boldsymbol{\gamma}(\mathbf{u}), \mathbf{0}, 0) d s+\int_{\Gamma_{S}}\left(h_{S}^{*}+I_{\mathcal{K}}\right)^{*}(x,-\boldsymbol{\nu} \otimes \gamma(\mathbf{u}), \mathbf{0}, 0) d s \\
&+ \int_{\Gamma_{W}}\left(h_{W}^{*}+I_{\mathcal{K}}\right)^{*}(x,-\boldsymbol{\nu} \otimes \gamma(\mathbf{u}), \mathbf{0}, 0) d s+\int_{\Omega} j\left(x,(\nabla \mathbf{u})_{a}, \widetilde{\boldsymbol{\mu}}_{a}, \mu_{a}\right) d x \\
&+ \int_{\Omega} j_{\infty}\left(x, \frac{d(\nabla \mathbf{u})_{s}}{d\left|\left(\nabla \mathbf{u}_{s}, \widetilde{\boldsymbol{\mu}}_{s}, \mu_{s}\right)\right|}, \frac{d \widetilde{\boldsymbol{\mu}}_{s}}{d\left|\left(\nabla \mathbf{u}_{s}, \widetilde{\boldsymbol{\mu}}_{s}, \mu_{s}\right)\right|},\right. \\
&\left.\frac{d \mu_{s}}{d\left|\left(\nabla \mathbf{u}_{s}, \widetilde{\boldsymbol{\mu}}_{s}, \mu_{s}\right)\right|}\right) d\left|\left(\nabla \mathbf{u}_{s}, \widetilde{\boldsymbol{\mu}}_{s}, \mu_{s}\right)\right|
\end{aligned}
$$

for every $(\mathbf{u}, \tilde{\boldsymbol{\mu}}, \mu) \in P_{q}^{3} B V(\Omega)$ and every $q>0$, where $\tilde{\boldsymbol{\mu}}=\widetilde{\boldsymbol{\mu}}_{a} d x+\widetilde{\boldsymbol{\mu}}_{s}$, $\mu=\mu_{a} d x+\mu_{s}$ and $\nabla \mathbf{u}=(\nabla \mathbf{u})_{a} d x+(\nabla \mathbf{u})_{s}$ are the Lebesgue decompositions of $\widetilde{\boldsymbol{\mu}}, \mu$ and $\nabla \mathbf{u}$ into absolutely continuous and singular parts with respect to $d x$. Here $\left|\left(\nabla \mathbf{u}_{s}, \widetilde{\boldsymbol{\mu}}_{s}, \mu_{s}\right)\right|$ is the total variation measure associated with $\left(\nabla \mathbf{u}_{s}, \widetilde{\boldsymbol{\mu}}_{s}, \mu_{s}\right)$, i.e. for every $\left(\nabla \mathbf{u}_{s}, \widetilde{\boldsymbol{\mu}}_{s}, \mu_{s}\right)$-measurable subset $\widetilde{\Omega}$ of $\Omega$ we have 


$$
\begin{array}{r}
\left|\left(\nabla \mathbf{u}_{s}, \widetilde{\boldsymbol{\mu}}_{s}, \mu_{s}\right)\right|(\widetilde{\Omega})=\sup \left\{\int_{\widetilde{\Omega}} \boldsymbol{\varphi}: \nabla \mathbf{u}_{s}+\int_{\widetilde{\Omega}} \widetilde{\phi}: \widetilde{\boldsymbol{\mu}}_{s}+\int_{\widetilde{\Omega}} \phi \mu_{s} \mid\right. \\
\boldsymbol{\varphi}, \widetilde{\boldsymbol{\phi}} \in C_{0}\left(\Omega, \mathbb{E}^{3}\right), \phi \in C_{0}(\Omega, \mathbb{R}), \max _{i, j}\left(\left\|\varphi_{i j}\right\|_{C(\Omega)}\right) \leq 1, \\
\left.\max _{i, j}\left(\left\|\widetilde{\phi}_{i j}\right\|_{C(\Omega)}\right) \leq 1,\|\phi\|_{C(\Omega)} \leq 1\right\} .
\end{array}
$$

The function $\left(h_{S}^{*}+I_{\mathcal{K}}\right)^{*}: \Omega \times \mathbb{E}^{3} \times \mathbb{E}^{3} \times \mathbb{R} \rightarrow \mathbb{R} \cup\{\infty\}$ is given by

$$
\begin{array}{r}
\left(h_{S}^{*}+I_{\mathcal{K}}\right)^{*}(x,-\boldsymbol{\nu} \otimes \boldsymbol{\gamma}(\mathbf{u}), \mathbf{0}, 0)=\sup \left\{-\boldsymbol{\omega}^{0}:(\boldsymbol{\nu} \otimes \boldsymbol{\gamma}(\mathbf{u}))-h_{S}^{*}\left(x, \boldsymbol{\omega}^{0}\right) \mid\right. \\
\left.\left(\boldsymbol{\omega}^{0}, \mathbf{0}, 0\right) \in \mathcal{K}(x) \subset \mathbb{E}^{3} \times \mathbb{E}^{3} \times \mathbb{R}\right\}
\end{array}
$$

for every $\mathbf{u} \in B V$ and ds-a.e. $x \in \Gamma_{S}$ (see (2.22)). Similarly, the function $\left(h_{W}^{*}+I_{\mathcal{K}}\right)^{*}: \Omega \times \mathbb{E}^{3} \times \mathbb{E}^{3} \times \mathbb{R} \rightarrow \mathbb{R} \cup\{\infty\}$ is given by

$$
\begin{array}{r}
\left(h_{W}^{*}+I_{\mathcal{K}}\right)^{*}(x,-\boldsymbol{\nu} \otimes \boldsymbol{\gamma}(\mathbf{u}), \mathbf{0}, 0)=\sup \left\{-\boldsymbol{\omega}^{0}:(\boldsymbol{\nu} \otimes \boldsymbol{\gamma}(\mathbf{u}))-h_{W}^{*}\left(x, \boldsymbol{\omega}^{0}\right) \mid\right. \\
\left.\left(\boldsymbol{\omega}^{0}, \mathbf{0}, 0\right) \in \mathcal{K}(x) \subset \mathbb{E}^{3} \times \mathbb{E}^{3} \times \mathbb{R}\right\}
\end{array}
$$

for every $\mathbf{u} \in B V$ and ds-a.e. $x \in \Gamma_{W}$ (cf. (2.23)). Moreover, $\widetilde{F}^{* *}$ is l.s.c. in the topology (2.18)-(2.19).

REMARK 2. Under assumption (2.27) we can find an explicit form of $\left(h_{S}^{*}+I_{\mathcal{K}}\right)^{*}$ and $\left(h_{W}^{*}+I_{\mathcal{K}}\right)^{*}$ in $(2.24)$ (see $(2.29)$ and $\left.(2.31)\right)$. Indeed, let

$$
\left\{\left(\boldsymbol{\omega}^{0}, \mathbf{0}, 0\right) \in \mathbb{E}^{3} \times \mathbb{E}^{3} \times \mathbb{R} \mid \operatorname{tr} \boldsymbol{\omega}^{0} \leq 0,\left(\boldsymbol{\omega}^{0}\right)^{D}=\mathbf{0}\right\} \subset \mathcal{K}(x)
$$

where $\left(\boldsymbol{\omega}^{0}\right)^{D}$ is the deviator part of $\boldsymbol{\omega}^{0}$ (cf. (2.21)). Then by (2.21) and (2.22) we obtain

$$
A_{L} \subset A(x) \cap\left\{\boldsymbol{\omega}^{0} \mid\left(\boldsymbol{\omega}^{0}, \mathbf{0}, 0\right) \in \mathcal{K}(x)\right\} \subset A(x)
$$

and

$$
\begin{aligned}
h_{S}(x,-\boldsymbol{\nu} \otimes \mathbf{u}) & =\sup \left\{-\boldsymbol{\omega}^{0}:(\boldsymbol{\nu} \otimes \mathbf{u}) \mid \boldsymbol{\omega}^{0} \in A_{L}\right\} \\
& \leq\left(h_{S}^{*}+I_{\mathcal{K}}\right)^{*}(x,-\boldsymbol{\nu} \otimes \mathbf{u}, \mathbf{0}, 0) \leq h_{S}^{* *}(x,-\boldsymbol{\nu} \otimes \mathbf{u})
\end{aligned}
$$

for $d s$-a.e. $x \in \Gamma_{S}$ and every $\mathbf{u} \in \mathbb{R}^{3}$. Because $h_{S} \geq h_{S}^{* *}$ we have

$$
h_{S}(x,-\boldsymbol{\nu} \otimes \mathbf{u})=\left(h_{S}^{*}+I_{\mathcal{K}}\right)^{*}(x,-\boldsymbol{\nu} \otimes \mathbf{u}, \mathbf{0}, 0)=h_{S}^{* *}(x,-\boldsymbol{\nu} \otimes \mathbf{u})
$$

for $d s$-a.e. $x \in \Gamma_{S}$ and every $\mathbf{u} \in \mathbb{R}^{3}$.

In a similar way, by (2.23), we get

$$
h_{W}^{*}\left(x, \boldsymbol{\omega}^{0}\right)+I_{A_{L}}\left(\boldsymbol{\omega}^{0}\right) \geq h_{W}^{*}\left(x, \boldsymbol{\omega}^{0}\right)+I_{\left\{\boldsymbol{\omega}^{0} \mid\left(\boldsymbol{\omega}^{0}, \mathbf{0}, 0\right) \in \mathcal{K}(x)\right\}}\left(\boldsymbol{\omega}^{0}\right) \geq h_{W}^{*}\left(x, \boldsymbol{\omega}^{0}\right)
$$

for $d s$-a.e. $x \in \Gamma_{W}$, every $\omega^{0} \in \mathbb{E}^{3}$, and

$$
\begin{aligned}
h_{W}(x,-\boldsymbol{\nu} \otimes \mathbf{u}) & =\sup \left\{-\boldsymbol{\omega}^{0}:(\boldsymbol{\nu} \otimes \mathbf{u})-h_{W}^{*}\left(x, \boldsymbol{\omega}^{0}\right) \mid \boldsymbol{\omega}^{0} \in A_{L}\right\} \\
& \leq\left(h_{W}^{*}+I_{\mathcal{K}}\right)^{*}(x,-\boldsymbol{\nu} \otimes \mathbf{u}, \mathbf{0}, 0) \leq h_{W}^{* *}(x,-\boldsymbol{\nu} \otimes \mathbf{u})
\end{aligned}
$$

for $d s$-a.e. $x \in \Gamma_{W}$ and every $\mathbf{u} \in \mathbb{R}^{3}$. Because $h_{W} \geq h_{W}^{* *}$ we have 
for $d s$-a.e. $x \in \Gamma_{W}$ and every $\mathbf{u} \in \mathbb{R}^{3}$.

REMARK 3. We do not prove in this paper that $\widetilde{F}^{* *}$ is the largest l.s.c. minorant (in the topology (2.18)-(2.19)) less than $F$. We find only the bidual functional $\widetilde{F}^{* *}$ to the extension $\widetilde{F}$ of $F$.

Definition 6 . We say that $\widetilde{F}^{* *}$ is coercive if the following condition holds:

$$
\text { if }\left\|\left(\mathbf{u}_{m}, \tilde{\boldsymbol{\mu}}_{m}, \mu_{m}\right)\right\|_{P_{q}^{3} B V} \rightarrow \infty \text { then } \widetilde{F}^{* *}\left(\nabla \mathbf{u}_{m}, \widetilde{\boldsymbol{\mu}}_{m}, \mu_{m}\right) \rightarrow \infty
$$

for every $\left\{\left(\mathbf{u}_{m}, \tilde{\boldsymbol{\mu}}_{m}, \mu_{m}\right)\right\}_{m \in \mathbb{N}} \subset \sum_{q=1}^{\infty} P_{q}^{3} B V(\Omega)$.

Theorem 3. Assume that $d s\left(\Gamma_{0}\right)>0$ and $\widetilde{F}^{* *}$ is coercive. Then there exist $q_{0}>0$ and $\left(\mathbf{u}_{0}, \widetilde{\boldsymbol{\mu}}_{0}, \mu_{0}\right) \in P_{q_{0}}^{3} B V(\Omega)$ such that

$$
\begin{aligned}
& \widetilde{F}^{* *}\left(\gamma\left(\mathbf{u}_{0}\right), \nabla \mathbf{u}_{0}, \widetilde{\boldsymbol{\mu}}_{0}, \mu_{0}\right) \\
& \quad=\inf \left\{\widetilde{F}^{* *}(\gamma(\mathbf{u}), \nabla \mathbf{u}, \widetilde{\boldsymbol{\mu}}, \mu) \mid(\mathbf{u}, \widetilde{\boldsymbol{\mu}}, \mu) \in \sum_{q=1}^{\infty} P_{q}^{3} B V(\Omega)\right\} .
\end{aligned}
$$

Below we study the properties of the space $P_{q}^{3} B V(\Omega)$ and we prove Theorems 2 and 3.

3. Relaxation of the unilateral contact conditions. Here we find the l.s.c. relaxation of our polyconvex functional with unilateral contact conditions. Moreover, we prove the existence theorem.

Definition 7. The sequence $\left\{\mathbf{u}_{n}\right\}_{n \in \mathbb{N}} \subset B V\left(\Omega, \mathbb{R}^{3}\right)$ converges to $\mathbf{u}^{0} \in$ $B V\left(\Omega, \mathbb{R}^{3}\right)$ in the topology (3.1) if

$$
\int_{\Omega} \boldsymbol{\sigma}:\left(\nabla \mathbf{u}_{n}-\nabla \mathbf{u}^{0}\right)-\int_{\partial \Omega} \boldsymbol{\sigma}:\left(\boldsymbol{\nu} \otimes \gamma\left(\mathbf{u}_{n}-\mathbf{u}^{0}\right)\right) d s \rightarrow 0
$$

for every $\boldsymbol{\sigma} \in C\left(\bar{\Omega}, \mathbb{E}^{3}\right)$.

Definition 8. The sequence $\left\{\left(\mathbf{u}_{n}, \widetilde{\boldsymbol{\mu}}_{n}, \mu_{n}\right)\right\}_{n \in \mathbb{N}} \subset P_{q}^{3} B V(\Omega) \subset$ $B V\left(\Omega, \mathbb{R}^{3}\right) \times \mathbb{M}_{b}\left(\Omega, \mathbb{E}^{3}\right) \times \mathbb{M}_{b}(\Omega, \mathbb{R})$ converges to $\left(\mathbf{u}^{0}, \widetilde{\boldsymbol{\mu}}^{0}, \mu^{0}\right) \in P_{q}^{3} B V(\Omega)$ in the topology (3.1)-(3.2) if

$$
\begin{cases}\widetilde{\boldsymbol{\mu}}_{n} \rightarrow \widetilde{\boldsymbol{\mu}}^{0} & \text { weak }^{*} \text { in } \mathbb{M}_{b}\left(\Omega, \mathbb{E}^{3}\right), \\ \mu_{n} \rightarrow \mu^{0} & \text { weak }{ }^{*} \text { in } \mathbb{M}_{b}(\Omega, \mathbb{R}),\end{cases}
$$

and $\left\{\mathbf{u}_{n}\right\}_{n \in \mathbb{N}}$ converges to $\mathbf{u}^{0}$ in the topology (3.1).

In [18] it was proved that a closed ball in $B V\left(\Omega, \mathbb{R}^{3}\right)$ is compact in the norm $\|\cdot\|_{L^{1}}$, or in $L_{\text {loc }}^{1}\left(\Omega, \mathbb{R}^{3}\right)$ topology.

Proposition 4 (cf. [5]). Every ball $\mathrm{cl}_{\|\cdot\|_{B V}} B_{B V}(0, \widehat{r})$ in $B V\left(\Omega, \mathbb{R}^{3}\right)$, closed in the norm $\|\cdot\|_{B V}$, is compact in the topology (3.1). 
Proof. We proceed in several steps.

Step 1. The space $B V\left(\Omega, \mathbb{R}^{3}\right)$ is isomorphic to

$$
A_{1} \equiv\left\{\mathbf{u} \in B V\left(\Omega_{1}, \mathbb{R}^{3}\right) \mid \mathbf{u}(x)=\mathbf{0} \text { for } d x \text {-a.e. } x \in \Omega_{1}-\bar{\Omega}\right\},
$$

the isomorphism being given by $A_{1} \ni \mathbf{u} \mapsto \mathbf{u}_{\mid \Omega} \in B V\left(\Omega, \mathbb{R}^{3}\right)$. We say that a sequence $\left\{\widetilde{\mathbf{u}}_{n}\right\}_{n \in \mathbb{N}} \subset B V\left(\Omega_{1}, \mathbb{R}^{3}\right)$ converges to $\widetilde{\mathbf{u}} \in B V\left(\Omega_{1}, \mathbb{R}^{3}\right)$ in the topology (3.4) if

$$
\begin{cases}\widetilde{\mathbf{u}}_{n} \rightarrow \widetilde{\mathbf{u}} & \text { in }\|\cdot\|_{L^{1}\left(\Omega_{1}, \mathbb{R}^{3}\right)}, \\ \nabla \widetilde{\mathbf{u}}_{n} \rightarrow \nabla \widetilde{\mathbf{u}} & \text { weak* in } \mathbb{M}_{b}\left(\Omega_{1}, \mathbb{E}^{3}\right) .\end{cases}
$$

We first show that the closed ball $\mathrm{cl}_{\|\cdot\|_{B V}} B_{B V\left(\Omega_{1}, \mathbb{R}^{3}\right)}(0, \widehat{r})$, endowed with the topology (3.4), is compact.

Indeed, since $\left\{\mathbf{u}_{n}\right\}_{n \in \mathbb{N}} \subset \operatorname{cl}_{\|\cdot\|_{B V}} B_{B V\left(\Omega_{1}, \mathbb{R}^{3}\right)}(0, \widehat{r})$, it follows that $\left\{\mathbf{u}_{n}\right\}_{n \in \mathbb{N}}$ $\subset \mathrm{cl}_{\|\cdot\|_{B D}} B_{B D\left(\Omega_{1}\right)}(0, \widehat{r})$ (see [23, pp. 143-145]). In view of the compactness theorem for $B D\left(\Omega_{1}\right)$ (see e.g. [23, Chapter 2, Theorem 2.4]) the injection of $\left[B D\left(\Omega_{1}\right),\|\cdot\|_{B D}\right]$ into $\left[L^{1}\left(\Omega_{1}, \mathbb{R}^{3}\right),\|\cdot\|_{L^{1}}\right]$ is compact. Hence, there exists a subsequence $\left\{\mathbf{u}_{n_{m}}\right\}_{m \in \mathbb{N}}$ of $\left\{\mathbf{u}_{n}\right\}_{n \in \mathbb{N}}$ and a field $\mathbf{u}_{0} \in L^{1}\left(\Omega_{1}, \mathbb{R}^{3}\right)$ such that $\mathbf{u}_{n_{m}} \rightarrow \mathbf{u}_{0}$ in $\|\cdot\|_{L^{1}\left(\Omega_{1}, \mathbb{R}^{3}\right)}$.

Step 2. By [10, Theorem V.5.1], a closed ball in $\mathbb{M}_{b}(\bar{\Omega}, \mathbb{R})$, endowed with the weak* topology, is metrizable.

Indeed, let $\left\{\varphi_{m}\right\}_{m \in \mathbb{N}}$ be a countable dense subset of $\left[C_{0}(\Omega, \mathbb{R}),\|\cdot\|_{C}\right]$ and

$$
\varrho\left(\mu_{1}, \mu_{2}\right)=\sum_{m=1}^{\infty} \frac{1}{2^{m}} \cdot \frac{\left|\int_{\Omega} \varphi_{m}\left(\mu_{1}-\mu_{2}\right)\right|}{1+\left|\int_{\Omega} \varphi_{m}\left(\mu_{1}-\mu_{2}\right)\right|}
$$

for $\mu_{1}, \mu_{2} \in \mathbb{M}_{b}(\Omega, \mathbb{R})$. It is easy to verify that $\varrho$ is a metric, since $\left\{\varphi_{m}\right\}_{m \in \mathbb{N}}$ is dense in $C_{0}(\Omega, \mathbb{R})$. The topology induced by $\varrho$ is weaker than the weak* $\mathbb{M}_{b}(\Omega, \mathbb{R})$ topology on $\mathrm{cl}_{\|\cdot\|_{\mathbb{M}_{b}}} B_{\mathbb{M}_{b}(\Omega, \mathbb{R})}(0, q)$. Since the other set endowed with the weak ${ }^{*} \mathbb{M}_{b}(\Omega, \mathbb{R})$ topology is compact, the topology induced by $\varrho$ on it coincides with the weak* $\mathbb{M}_{b}(\Omega, \mathbb{R})$ topology (cf. [13, Corollary 3.1.14]).

Step 3. The sequence $\left\{\nabla \mathbf{u}_{n_{m}}\right\}_{m \in \mathbb{N}}$ is bounded in $\|\cdot\|_{\mathbb{M}_{b}\left(\Omega_{1}, \mathbb{E}^{3}\right)}$. By [10, Theorem V.5.1], $\mathrm{cl}_{\|\cdot\|_{\mathbb{M}_{b}}} B_{\mathbb{M}_{b}\left(\Omega_{1}, \mathbb{E}^{3}\right)}(0, \widehat{r})$ endowed with the weak* topology is metrizable (see Section 2). Since it is compact, there exists a subsequence $\left\{\nabla \mathbf{u}_{n_{m_{p}}}\right\}_{p \in \mathbb{N}}$ and $\tilde{\boldsymbol{\mu}} \in \mathbb{M}_{b}\left(\Omega_{1}, \mathbb{E}^{3}\right)$ such that

$$
\nabla \mathbf{u}_{n_{m_{p}}} \rightarrow \widetilde{\boldsymbol{\mu}} \quad \text { weak }^{*} \text { in } \mathbb{M}_{b}\left(\Omega_{1}, \mathbb{E}^{3}\right) .
$$

Moreover, $\widetilde{\boldsymbol{\mu}}=\nabla \mathbf{u}_{0}$ in the distributional sense, because

$$
\int_{\Omega_{1}} \boldsymbol{\sigma}: \nabla \mathbf{u}_{n_{m_{p}}}=-\int_{\Omega_{1}}(\operatorname{div} \boldsymbol{\sigma}) \cdot \mathbf{u}_{n_{m_{p}}} d x \quad \forall \boldsymbol{\sigma} \in C_{c}^{1}\left(\Omega_{1}, \mathbb{E}^{3}\right) .
$$

Step 4. In this step, we prove that $\left\|\mathbf{u}_{0}\right\|_{B V\left(\Omega_{1}, \mathbb{R}^{3}\right)} \leq \widehat{r}$. Indeed, by Step 1 ,

$$
\lim \left\|\mathbf{u}_{n_{m_{p}}}\right\|_{L^{1}\left(\Omega_{1}, \mathbb{R}^{3}\right)}=\left\|\mathbf{u}_{0}\right\|_{L^{1}\left(\Omega_{1}, \mathbb{R}^{3}\right)} .
$$


Thus for every $\delta>0$, there exists $p_{\delta} \in \mathbb{N}$ such that for every $p>p_{\delta}$,

$$
\begin{aligned}
& \widehat{r}-\lim \left\|\mathbf{u}_{n_{m_{p}}}\right\|_{L^{1}}+\delta \geq \sup \left\{\left\|\nabla \mathbf{u}_{n_{m_{p}}}\right\|_{\mathbb{M}_{b}\left(\Omega_{1}, \mathbb{E}^{3}\right)} \mid p \in \mathbb{N}, p>p_{\delta}\right\} \\
& =\sup _{p>p_{\delta}} \sup \left\{\int_{\Omega_{1}} \boldsymbol{\omega}: \nabla \mathbf{u}_{n_{m_{p}}} \mid \boldsymbol{\omega} \in C_{0}\left(\Omega_{1}, \mathbb{E}^{3}\right), \max _{i, j}\left\|\omega_{i j}\right\|_{C_{0}\left(\Omega_{1}\right)} \leq 1\right\} \\
& =\sup \left\{\sup _{p>p_{\delta}} \int_{\Omega_{1}} \boldsymbol{\omega}: \nabla \mathbf{u}_{n_{m_{p}}} \mid \boldsymbol{\omega} \in C_{0}\left(\Omega_{1}, \mathbb{E}^{3}\right), \max _{i, j}\left\|\omega_{i j}\right\|_{C_{0}\left(\Omega_{1}\right)} \leq 1\right\} \\
& \geq \sup \left\{\lim _{p} \int_{\Omega_{1}} \boldsymbol{\omega}: \nabla \mathbf{u}_{n_{m_{p}}} \mid \boldsymbol{\omega} \in C_{0}\left(\Omega_{1}, \mathbb{E}^{3}\right), \max _{i, j}\left\|\omega_{i j}\right\|_{C_{0}\left(\Omega_{1}\right)} \leq 1\right\} \\
& =\sup \left\{\int_{\Omega_{1}} \boldsymbol{\omega}: \nabla \mathbf{u}_{0} \mid \boldsymbol{\omega} \in C_{0}\left(\Omega_{1}, \mathbb{E}^{3}\right), \max _{i, j}\left\|\omega_{i j}\right\|_{C_{0}\left(\Omega_{1}\right)} \leq 1\right\} \\
& =\sum_{i, j=1}^{3}\left\|\frac{\partial\left(u_{0}\right)_{i}}{\partial x_{j}}\right\|_{\mathbb{M}_{b}\left(\Omega_{1}, \mathbb{R}\right)}=\left\|\nabla \mathbf{u}_{0}\right\|_{\mathbb{M}_{b}\left(\Omega_{1}, \mathbb{E}^{3}\right)}
\end{aligned}
$$

(cf. (2.1) and the definition of $\|\cdot\|_{\mathbb{E}^{3}}$ ). Hence we have

$$
\begin{aligned}
\widehat{r}+\delta & \geq \lim \left\|\mathbf{u}_{n_{m_{p}}}\right\|_{L^{1}\left(\Omega_{1}, \mathbb{R}^{3}\right)}+\left\|\nabla \mathbf{u}_{0}\right\|_{\mathbb{M}_{b}\left(\Omega_{1}, \mathbb{E}^{3}\right)} \\
& \geq\left\|\mathbf{u}_{0}\right\|_{L^{1}\left(\Omega_{1}, \mathbb{R}^{3}\right)}+\left\|\nabla \mathbf{u}_{0}\right\|_{\mathbb{M}_{b}\left(\Omega_{1}, \mathbb{E}^{3}\right)}
\end{aligned}
$$

for every $\delta>0$.

Step 5. Let $\left\{\mathbf{u}_{n}\right\}_{n \in \mathbb{N}} \subset \operatorname{cl}_{\|\cdot\|_{B V}} B_{B V\left(\Omega, \mathbb{R}^{3}\right)}(0, \widehat{r}) \subset B V\left(\Omega, \mathbb{R}^{3}\right)$ and let $\left\{\widehat{\mathbf{u}}_{n}\right\}_{n \in \mathbb{N}} \subset B V\left(\Omega_{1}, \mathbb{R}^{3}\right)$ be the extension of $\left\{\mathbf{u}_{n}\right\}_{n \in \mathbb{N}}$ to $\Omega_{1}$, given by

$$
\widehat{\mathbf{u}}_{n \mid \Omega}=\mathbf{u}_{n}, \quad \widehat{\mathbf{u}}_{n \mid \Omega_{1}-\bar{\Omega}}=\mathbf{0},
$$

for every $n \in \mathbb{N}$. By Step 1 there exists a subsequence $\left\{\widehat{\mathbf{u}}_{n_{m}}\right\}_{m \in \mathbb{N}}$ and $\widehat{\mathbf{u}} \in$ $B V\left(\Omega_{1}, \mathbb{R}^{3}\right)$ such that

$$
\begin{aligned}
& \widehat{\mathbf{u}}_{n_{m}} \rightarrow \widehat{\mathbf{u}} \quad \text { in }\|\cdot\|_{L^{1}\left(\Omega_{1}, \mathbb{R}^{3}\right)}, \\
& \nabla \widehat{\mathbf{u}}_{n_{m}} \rightarrow \nabla \widehat{\mathbf{u}} \quad \text { weak }^{*} \text { in } \mathbb{M}_{b}\left(\Omega_{1}, \mathbb{E}^{3}\right)
\end{aligned}
$$

and $\widehat{\mathbf{u}}_{\mid \Omega_{1}-\bar{\Omega}}=\mathbf{0}$ (cf. (3.4), (3.5)).

We say that a sequence $\left\{\overline{\mathbf{u}}_{n}\right\}_{n \in \mathbb{N}} \subset B V\left(\Omega, \mathbb{R}^{3}\right)$ converges to $\overline{\mathbf{u}}$ in the topology (3.9)-(3.10) if

$$
\begin{gathered}
\overline{\mathbf{u}}_{n} \rightarrow \overline{\mathbf{u}} \quad \text { in }\|\cdot\|_{L^{1}\left(\Omega, \mathbb{R}^{3}\right)}, \\
\int_{\Omega} \boldsymbol{\sigma}:\left(\nabla \overline{\mathbf{u}}_{n}-\nabla \overline{\mathbf{u}}\right)-\int_{\partial \Omega} \boldsymbol{\sigma}:\left(\boldsymbol{\nu} \otimes \gamma\left(\overline{\mathbf{u}}_{n}-\overline{\mathbf{u}}\right)\right) d s \rightarrow 0
\end{gathered}
$$

for all $\boldsymbol{\sigma} \in C\left(\bar{\Omega}, \mathbb{E}^{3}\right)$. Observe that

$$
\nabla \widehat{\mathbf{u}}_{n_{m}}=\nabla \widehat{\mathbf{u}}_{n_{m} \mid \Omega}+\boldsymbol{\nu} \otimes\left(\gamma\left(\widehat{\mathbf{u}}_{n_{m} \mid \Omega_{1}-\bar{\Omega}}\right)-\gamma\left(\widehat{\mathbf{u}}_{n_{m} \mid \Omega}\right)\right) d s+\nabla \widehat{\mathbf{u}}_{n_{m} \mid \Omega_{1}-\bar{\Omega}},
$$


where $\boldsymbol{\gamma}\left(\widehat{\mathbf{u}}_{n_{m} \mid \Omega}\right)$ and $\boldsymbol{\gamma}\left(\widehat{\mathbf{u}}_{n_{m} \mid \Omega_{1}-\bar{\Omega}}\right)$ respectively stand for the interior and exterior trace of $\widehat{\mathbf{u}}_{n_{m}}$ on $\partial \Omega$ (cf. [23], [24]). Hence, by (3.7)-(3.8), $\left\{\mathbf{u}_{n_{m}}\right\}_{m \in \mathbb{N}}$ converges to $\widehat{\mathbf{u}}_{\mid \Omega}$ in the topology (3.9)-(3.10). The sequence $\left\{\left(\nabla \mathbf{u}_{n_{m}}, \boldsymbol{\nu} \otimes\right.\right.$ $\left.\left.\gamma\left(\mathbf{u}_{n_{m}}\right)\right)\right\}_{m \in \mathbb{N}}$ of measures on $\bar{\Omega}$ is bounded in $\|\cdot\|_{\mathbb{M}_{b}\left(\bar{\Omega}, \mathbb{E}^{3}\right)}$, since $\left\{\mathbf{u}_{n}\right\}_{n \in \mathbb{N}} \subset$ $\mathrm{cl}_{\|\cdot\|_{B V}} B_{B V\left(\Omega, \mathbb{R}^{3}\right)}(0, \widehat{r})$ and $\gamma$ is a continuous trace from $\left[B V,\|\cdot\|_{B V}\right]$ to $\left[L^{1}(\partial \Omega),\|\cdot\|_{L^{1}}\right]$. By $\left[10\right.$, Theorem V.5.1], closed balls in $\mathbb{M}_{b}\left(\bar{\Omega}, \mathbb{E}^{3}\right)$, endowed with the weak* topology, are metrizable (see Step 2). Thus $\mathrm{cl}_{\|\cdot\|_{B V}} B_{B V\left(\Omega, \mathbb{R}^{3}\right)}(0, \widehat{r})$, endowed with the topology (3.9)-(3.10), is metrizable, and hence compact (see also Step 4). Notice that the topology (3.1) is weaker than (3.9)-(3.10), and moreover, (3.1) is a Hausdorff topology. It is known that, among all Hausdorff topologies, compact topologies are minimal (see [13, Corollary 3.1.14]). From these observations, it follows that the closed ball $\mathrm{cl}_{\|\cdot\|_{B V}} B_{B V\left(\Omega, \mathbb{R}^{3}\right)}(0, \widehat{r})$, endowed with the topology (3.1), is compact.

Definition 9. We say that a sequence $\left\{\mathbf{u}_{n}\right\}_{n \in \mathbb{N}} \subset B V\left(\Omega, \mathbb{R}^{3}\right)$ converges to $\mathbf{u}^{0} \in B V\left(\Omega, \mathbb{R}^{3}\right)$ in the topology (3.11) if

$$
\begin{cases}\mathbf{u}_{n} \rightarrow \mathbf{u}^{0} & \text { in }\|\cdot\|_{L^{1}\left(\Omega, \mathbb{R}^{3}\right)}, \\ \nabla \mathbf{u}_{n} \rightarrow \nabla \mathbf{u}^{0} & \text { weak }^{*} \text { in } \mathbb{M}_{b}\left(\Omega, \mathbb{E}^{3}\right) .\end{cases}
$$

Furthermore, a sequence $\left\{\left(\mathbf{u}_{n}, \widetilde{\boldsymbol{\mu}}_{n}, \mu_{n}\right)\right\}_{n \in \mathbb{N}} \subset P_{q}^{3} B V(\Omega) \subset B V\left(\Omega, \mathbb{R}^{3}\right) \times$ $\mathbb{M}_{b}\left(\Omega, \mathbb{E}^{3}\right) \times \mathbb{M}_{b}(\Omega, \mathbb{R})$ converges to $\left(\mathbf{u}^{0}, \widetilde{\boldsymbol{\mu}}^{0}, \mu^{0}\right) \in P_{q}^{3} B V(\Omega)$ in the topology (3.11)-(3.12) if

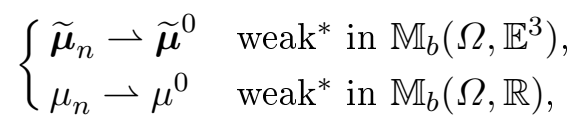

and $\left\{\mathbf{u}_{n}\right\}_{n \in \mathbb{N}}$ converges to $\mathbf{u}^{0}$ in the topology (3.11).

We obtain the following result.

Lemma 5 (cf. [5]). On every closed ball $\mathrm{cl}_{\|\cdot\|_{B V}} B_{B V\left(\Omega, \mathbb{R}^{3}\right)}(0, \widehat{r})$, the topology (3.1) is equivalent to the topology (3.11).

Proof. Consider a sequence $\left\{\mathbf{u}_{n}\right\}_{n \in \mathbb{N}} \subset \mathrm{cl}_{\|\cdot\|_{B V}} B_{B V\left(\Omega, \mathbb{R}^{3}\right)}(0, \widehat{r})$, and let $\left\{\widehat{\mathbf{u}}_{n}\right\}_{n \in \mathbb{N}} \subset B V\left(\Omega_{1}, \mathbb{R}^{3}\right)$ be the extension of $\left\{\mathbf{u}_{n}\right\}_{n \in \mathbb{N}}$ to $\Omega_{1}$, given by (3.6). As seen in the proof of Proposition 4 (Step 5), the convergence of $\left\{\mathbf{u}_{n}\right\}_{n \in \mathbb{N}}$ to $\mathbf{u}^{0} \in \operatorname{cl}_{\|\cdot\|_{B V}} B_{B V\left(\Omega, \mathbb{R}^{3}\right)}(0, \widehat{r})$ in the topology (3.1) is equivalent to the convergence of $\left\{\widehat{\mathbf{u}}_{n}\right\}_{n \in \mathbb{N}} \subset \mathrm{cl}_{\|\cdot\|_{B V}} B_{B V\left(\Omega_{1}, \mathbb{R}^{3}\right)}(0, \widehat{r})$ to $\widehat{\mathbf{u}}^{0} \in \mathrm{cl}_{\|\cdot\|_{B V}} B_{B V\left(\Omega_{1}, \mathbb{R}^{3}\right)}(0, \widehat{r})$ in the topology (3.4), where $\widehat{\mathbf{u}}_{\mid \Omega}^{0}=\mathbf{u}^{0}$ and $\widehat{\mathbf{u}}_{\mid \Omega_{1}-\bar{\Omega}}^{0}=\mathbf{0}$.

The set $A_{1} \cap \operatorname{cl}_{\|\cdot\|_{B V}} B_{B V\left(\Omega_{1}, \mathbb{R}^{3}\right)}(0, \widehat{r})$ endowed with the topology (3.4) is compact (see (3.3) and proof of Proposition 4). The Hausdorff topology 
on $A_{1}$, given by

$$
\begin{cases}\widehat{\mathbf{u}}_{n} \rightarrow \widehat{\mathbf{u}} & \text { in }\|\cdot\|_{L^{1}\left(\Omega_{1}, \mathbb{R}^{3}\right)}, \\ \int_{\Omega} \boldsymbol{\varphi}: \nabla \widehat{\mathbf{u}}_{n} \rightarrow \int_{\Omega} \boldsymbol{\varphi}: \nabla \widehat{\mathbf{u}} & \forall \boldsymbol{\varphi} \in C_{0}\left(\Omega, \mathbb{E}^{3}\right),\end{cases}
$$

is weaker than the topology (3.4) (cf. (3.3)). Thus, on

$$
A_{1} \cap \mathrm{cl}_{\|\cdot\|_{B V}} B_{B V\left(\Omega_{1}, \mathbb{R}^{3}\right)}(0, \widehat{r}),
$$

the topology (3.4) is equivalent to (3.13) (see [13, Corollary 3.1.14]). Moreover, the topology (3.13) on $A_{1}$ is equivalent to the topology (3.11) on $B V(\Omega)$, because $A_{1}$ is isomorphic to $B V\left(\Omega, \mathbb{R}^{3}\right)$.

Corollary 6. The topology (3.1)-(3.2) is equivalent to the topology (3.11)-(3.12) on closed balls in $B V\left(\Omega, \mathbb{R}^{3}\right) \times \mathbb{M}_{b}\left(\Omega, \mathbb{E}^{3}\right) \times \mathbb{M}_{b}(\Omega, \mathbb{R})$.

Theorem 7. The set $P_{q}^{3} B V(\Omega)$ endowed with the topology (3.11)-(3.12) or (3.1)-(3.2)] is compact.

Proof. We proceed in several steps.

Step 1. In view of Definition 4 , we have

$$
\begin{aligned}
P_{q}^{3} B V(\Omega) \subset & \operatorname{cl}_{\|\cdot\|_{B V}} B_{B V\left(\Omega, \mathbb{R}^{3}\right)}(0, q) \\
& \times \operatorname{cl}_{\|\cdot\|_{\mathbb{M}_{b}}} B_{\mathbb{M}_{b}\left(\Omega, \mathbb{E}^{3}\right)}(0, q) \times \operatorname{cl}_{\|\cdot\|_{\mathbb{M}_{b}}} B_{\mathbb{M}_{b}(\Omega, \mathbb{R})}(0, q) .
\end{aligned}
$$

Next, we argue that the closed ball $B \equiv \operatorname{cl}_{\|\cdot\|_{B V}} B_{B V\left(\Omega, \mathbb{R}^{3}\right)}(0, q)$ endowed with the topology (3.11) is metrizable.

Indeed, $B$ endowed with $\|\cdot\|_{L^{1}}$ is a Hausdorff topological space. Moreover, the topology induced by $\|\cdot\|_{L^{1}}$ is weaker than (3.11). Since $B$ endowed with the topology (3.11) is compact, this topology coincides with the topology induced by $\|\cdot\|_{L^{1}}$ on $B$ (cf. Proposition 4 , Lemma 5 and [13, Corollary 3.1.14]).

Step 2. By Lemma 5 and the previous step, $B$ endowed with the topology (3.1) is metrizable.

Step 3. By [10, Theorem V.5.1], the closed ball $\mathrm{cl}_{\|\cdot\|_{\mathbb{M}_{b}}} B_{\mathbb{M}_{b}(\Omega, \mathbb{R})}(0, q)$ endowed with the weak* topology is also a metrizable topological space (see proof of Proposition 4, Step 2). Similarly, by [10, Theorem V.5.1], a closed bounded ball in $\mathbb{M}_{b}\left(\bar{\Omega}, \mathbb{E}^{3}\right)$, endowed with the weak* topology, is metrizable.

Step 4. On account of Steps 3 and 4 and of Lemma 5, the set $P_{q}^{3} B V(\Omega)$ endowed with the topology (3.11)-(3.12) (or the topology (3.1)-(3.2)) is metrizable (cf. Definition 4 and (3.14)). Then we consider a sequence

$$
\left\{\left(\mathbf{u}_{m}, \tilde{\boldsymbol{\mu}}_{m}, \mu_{m}\right)\right\}_{m \in \mathbb{N}} \subset P_{q}^{3} B V(\Omega) .
$$


By (3.14) and Proposition 4, there is a subsequence $\left\{\mathbf{u}_{m_{p}}\right\}_{p \in \mathbb{N}} \subset B V\left(\Omega, \mathbb{R}^{3}\right)$ and $\mathbf{u}_{0} \in B V\left(\Omega, \mathbb{R}^{3}\right)$ such that

$$
\mathbf{u}_{m_{p}} \rightarrow \mathbf{u}_{0} \quad \text { in the topology (3.1) (or (3.11)). }
$$

Moreover, by Proposition 4, for every $\widetilde{\delta}>0$ we obtain

$$
\left\|\mathbf{u}_{0}\right\|_{B V} \leq \liminf \left\{\left\|\mathbf{u}_{m_{p}}\right\|_{B V} \mid p \in \mathbb{N}\right\}+\widetilde{\delta},
$$

because there exists a subsequence $\left\{\mathbf{u}_{m_{p_{t}}}\right\}_{t \in \mathbb{N}}$ such that $\mathbf{u}_{m_{p_{t}}} \rightarrow \mathbf{u}_{0}$ in the topology (3.1) and $\left\{\mathbf{u}_{m_{p_{t}}}\right\}_{t \in \mathbb{N}} \subset B_{B V}\left(0, \liminf \left\|\mathbf{u}_{m_{p}}\right\|_{B V}+\widetilde{\delta}\right)$.

Step 5. We consider again the sequence $\left\{\left(\mathbf{u}_{m}, \widetilde{\boldsymbol{\mu}}_{m}, \mu_{m}\right)\right\}_{m \in \mathbb{N}} \subset P_{q}^{3} B V(\Omega)$, defined in Step 4. There exist a subsequence $\left\{\left(\widetilde{\boldsymbol{\mu}}_{m_{p_{t}}}, \mu_{m_{p_{t}}}\right)\right\}_{t \in \mathbb{N}} \subset \mathbb{M}_{b}\left(\Omega, \mathbb{E}^{3}\right)$ $\times \mathbb{M}_{b}(\Omega, \mathbb{R})$ and $\left(\widetilde{\boldsymbol{\mu}}_{0}, \mu_{0}\right) \in \mathbb{M}_{b}\left(\Omega, \mathbb{E}^{3}\right) \times \mathbb{M}_{b}(\Omega, \mathbb{R})$ such that

$$
\begin{gathered}
\widetilde{\boldsymbol{\mu}}_{m_{p_{t}}} \rightarrow \widetilde{\boldsymbol{\mu}}_{0} \quad \text { weak }^{*} \text { in } \mathbb{M}_{b}\left(\Omega, \mathbb{E}^{3}\right), \\
\mu_{m_{p_{t}}} \rightarrow \mu_{0} \quad \text { weak }^{*} \text { in } \mathbb{M}_{b}(\Omega, \mathbb{R}) .
\end{gathered}
$$

Moreover, we can assume that for every $\widetilde{\delta}>0$, there exists $t_{\widetilde{\delta}} \in \mathbb{N}$ such that

$$
\begin{aligned}
q-\liminf \{ & \left\{\mathbf{u}_{m_{p}} \|_{B V} \mid p \in \mathbb{N}\right\}+\widetilde{\delta} \\
& \geq \sup \left\{\left\|\widetilde{\boldsymbol{\mu}}_{m_{p_{t}}}\right\|_{\mathbb{M}_{b}\left(\Omega, \mathbb{E}^{3}\right)}+\left\|\mu_{m_{p_{t}}}\right\|_{\mathbb{M}_{b}(\Omega)} \mid t \in \mathbb{N}, t>t_{\widetilde{\delta}}\right\}
\end{aligned}
$$

(cf. (2.8) and Definition 4). Similarly to Step 4 in the proof of Proposition 4, we obtain

$$
\begin{aligned}
& \sup \left\{\left\|\widetilde{\boldsymbol{\mu}}_{m_{p_{t}}}\right\|_{\mathbb{M}_{b}\left(\Omega, \mathbb{E}^{3}\right)}+\left\|\mu_{m_{p_{t}}}\right\|_{\mathbb{M}_{b}(\Omega)} \mid t \in \mathbb{N}, t>t_{\tilde{\delta}}\right\} \\
& =\sup _{t>t_{\tilde{\delta}}} \sup \left\{\int_{\Omega} \widetilde{\boldsymbol{\omega}}: \widetilde{\boldsymbol{\mu}}_{m_{p_{t}}}+\int_{\Omega} \omega \mu_{m_{p_{t}}} \mid\right. \\
& \left.\widetilde{\boldsymbol{\omega}} \in C_{0}\left(\Omega, \mathbb{E}^{3}\right), \omega \in C_{0}(\Omega, \mathbb{R}), \max _{i, j}\left(\left\|\widetilde{\omega}_{i j}\right\|_{C_{0}(\Omega)}\right) \leq 1,\|\omega\|_{C_{0}(\Omega)} \leq 1\right\} \\
& \geq \sup \left\{\lim _{t>t_{\widetilde{\delta}}}\left(\int_{\Omega} \widetilde{\boldsymbol{\omega}}: \widetilde{\boldsymbol{\mu}}_{m_{p_{t}}}+\int_{\Omega} \omega \mu_{m_{p_{t}}}\right) \mid\right. \\
& \left.\widetilde{\boldsymbol{\omega}} \in C_{0}\left(\Omega, \mathbb{E}^{3}\right), \omega \in C_{0}(\Omega, \mathbb{R}), \max _{i, j}\left(\left\|\widetilde{\omega}_{i j}\right\|_{C_{0}(\Omega)}\right) \leq 1,\|\omega\|_{C_{0}(\Omega)} \leq 1\right\} \\
& =\sup \left\{\int_{\Omega} \widetilde{\boldsymbol{\omega}}: \widetilde{\boldsymbol{\mu}}_{0}+\int_{\Omega} \omega \mu_{0} \mid \quad=\left\|\widetilde{\boldsymbol{\mu}}_{0}\right\|_{\mathbb{M}_{b}\left(\Omega, \mathbb{E}^{3}\right)}+\left\|\mu_{0}\right\|_{\mathbb{M}_{b}(\Omega, \mathbb{R})},\right. \\
& \widetilde{\boldsymbol{\omega}} \in C_{0}\left(\Omega, \mathbb{E}^{3}\right), \omega \in C_{0}(\Omega, \mathbb{R}), \max _{i, j}\left(\left\|\widetilde{\omega}_{i j}\right\|_{C_{0}(\Omega)} \leq 1,\|\omega\|_{C_{0}(\Omega)} \leq 1\right\}
\end{aligned}
$$

where $\widetilde{\omega}_{i j}$ are the components of $\widetilde{\boldsymbol{\omega}}$ and $i, j \in\{1,2,3\}$. Then, by (3.15)-(3.17) we get

$$
q+2 \widetilde{\delta} \geq\left\|\mathbf{u}_{0}\right\|_{B V\left(\Omega, \mathbb{R}^{3}\right)}+\left\|\widetilde{\boldsymbol{\mu}}_{0}\right\|_{\mathbb{M}_{b}\left(\Omega, \mathbb{E}^{3}\right)}+\left\|\mu_{0}\right\|_{\mathbb{M}_{b}(\Omega, \mathbb{R})}
$$


for every $\widetilde{\delta}>0$. Hence, we obtain

$$
\begin{aligned}
q & \geq\left\|\mathbf{u}_{0}\right\|_{B V\left(\Omega, \mathbb{R}^{3}\right)}+\left\|\widetilde{\boldsymbol{\mu}}_{0}\right\|_{\mathbb{M}_{b}\left(\Omega, \mathbb{E}^{3}\right)}+\left\|\mu_{0}\right\|_{\mathbb{M}_{b}(\Omega, \mathbb{R})} \\
& =\left\|\left(\mathbf{u}_{0}, \widetilde{\boldsymbol{\mu}}_{0}, \mu_{0}\right)\right\|_{P^{3} B V}
\end{aligned}
$$

(see also (2.8)).

Step 6. Finally, we show that $\left(\mathbf{u}_{0}, \widetilde{\boldsymbol{\mu}}_{0}, \mu_{0}\right) \in P_{q}^{3} B V(\Omega)$. To this end, notice that for every $t \in \mathbb{N}$ there exists a sequence $\left\{\mathbf{u}_{m_{p_{t}}}^{\tau}\right\}_{\tau \in \mathbb{N}} \subset C^{1}\left(\bar{\Omega}, \mathbb{R}^{3}\right)$ such that

$$
\begin{aligned}
\left\|\left(\mathbf{u}_{m_{p_{t}}}^{\tau}, \operatorname{Adj} \nabla \mathbf{u}_{m_{p_{t}}}^{\tau}, \operatorname{det}\left(\mathbf{u}_{m_{p_{t}}}^{\tau}\right)\right)\right\|_{P^{3} B V} \leq q & & \forall \tau \in \mathbb{N}, \\
\left\|\mathbf{u}_{m_{p_{t}}}^{\tau}-\mathbf{u}_{m_{p_{t}}}\right\|_{L^{1}\left(\Omega, \mathbb{R}^{3}\right)} \rightarrow 0 & & \text { as } \tau \rightarrow \infty,
\end{aligned}
$$

and moreover

$$
\begin{aligned}
& \nabla \mathbf{u}_{m_{p_{t}}}^{\tau} \rightarrow \nabla \mathbf{u}_{m_{p_{t}}} \quad \text { weak }^{*} \text { in } \mathbb{M}_{b}\left(\Omega, \mathbb{E}^{3}\right) \text { as } \tau \rightarrow \infty, \\
& \operatorname{Adj}\left(\nabla \mathbf{u}_{m_{p_{t}}}^{\tau}\right) \rightarrow \widetilde{\boldsymbol{\mu}}_{m_{p_{t}}} \quad \text { weak }^{*} \text { in } \mathbb{M}_{b}\left(\Omega, \mathbb{E}^{3}\right) \text { as } \tau \rightarrow \infty \text {, } \\
& \operatorname{det} \nabla \mathbf{u}_{m_{p_{t}}}^{\tau} \rightarrow \mu_{m_{p_{t}}} \quad \text { weak* }^{*} \text { in } \mathbb{M}_{b}(\Omega, \mathbb{R}) \text { as } \tau \rightarrow \infty
\end{aligned}
$$

(cf. Definition 4). Since $P_{q}^{3} B V(\Omega)$ endowed with the topology (3.11)-(3.12) is metrizable and for every $\tau, t \in \mathbb{N}$,

$$
\left(\mathbf{u}_{m_{p_{t}}}^{\tau}, \operatorname{Adj} \nabla \mathbf{u}_{m_{p_{t}}}^{\tau}, \operatorname{det}\left(\nabla \mathbf{u}_{m_{p_{t}}}^{\tau}\right)\right) \in P_{q}^{3} B V(\Omega),
$$

there exists a sequence $\left\{\mathbf{u}_{m_{p_{t_{k}}}}^{\tau_{k}}\right\}_{k \in \mathbb{N}}$ in $C^{1}\left(\bar{\Omega}, \mathbb{R}^{3}\right)$ such that

$$
\begin{aligned}
\left\|\left(\mathbf{u}_{m_{p_{k}}}^{\tau_{k}}, \operatorname{Adj} \nabla \mathbf{u}_{m_{p_{t_{k}}}}^{\tau_{k}}, \operatorname{det}\left(\nabla \mathbf{u}_{m_{p_{t_{k}}}}^{\tau_{k}}\right)\right)\right\|_{P^{3} B V} \leq q & \forall k \in \mathbb{N}, \\
\left\|\mathbf{u}_{m_{p_{t_{k}}}}^{\tau_{k}}-\mathbf{u}_{0}\right\|_{L^{1}\left(\Omega, \mathbb{R}^{3}\right)} \rightarrow 0 & \text { as } k \rightarrow \infty,
\end{aligned}
$$

and moreover

$$
\begin{aligned}
& \nabla \mathbf{u}_{m_{p_{t_{k}}}}^{\tau_{k}} \rightarrow \nabla \mathbf{u}_{0} \quad \text { weak }{ }^{*} \text { in } \mathbb{M}_{b}\left(\Omega, \mathbb{E}^{3}\right) \text { as } k \rightarrow \infty, \\
& \operatorname{Adj} \nabla \mathbf{u}_{m_{p_{k}}}^{\tau_{k}} \rightarrow \widetilde{\boldsymbol{\mu}}_{0} \quad \text { weak }^{*} \text { in } \mathbb{M}_{b}\left(\Omega, \mathbb{E}^{3}\right) \text { as } k \rightarrow \infty \text {, } \\
& \operatorname{det} \nabla \mathbf{u}_{m_{p_{t_{k}}}}^{\tau_{k}} \rightarrow \mu_{0} \quad \text { weak }^{*} \text { in } \mathbb{M}_{b}(\Omega, \mathbb{R}) \text { as } k \rightarrow \infty \text {. }
\end{aligned}
$$

It follows, by Definition 4 and (3.18), that $\left(\mathbf{u}_{0}, \widetilde{\boldsymbol{\mu}}_{0}, \mu_{0}\right) \in P_{q}^{3} B V(\Omega)$.

Definition 10 (cf. [8]). A subset $H_{0}$ of $\mathcal{L}^{0}\left(\bar{\Omega}, \mathbb{R}^{m}\right)_{\mu}$ is said to be $P C U$ stable if for any continuous partition of unity $\left(\zeta_{0}, \ldots, \zeta_{d}\right)$ with $\zeta_{0}, \ldots, \zeta_{d} \in$ $C^{\infty}(\bar{\Omega}, \mathbb{R})$ and for every $\mathbf{z}_{0}, \ldots, \mathbf{z}_{d} \in H_{0}$, the sum $\sum_{i=0}^{d} \zeta_{i} \mathbf{z}_{i}$ is in $H_{0}$.

Proof of Theorem 2. The space $C^{1}\left(\bar{\Omega}, \mathbb{R}^{3}\right)$ is PCU-stable. Then, by $[8$, Theorem 1] and by [22, Theorem 3A and Proposition $2 \mathrm{M}]$, we have, for every $\left(\boldsymbol{\omega}^{0}, \boldsymbol{\omega}^{1}, \omega^{2}\right) \in C\left(\bar{\Omega}, \mathbb{E}^{3}\right) \times C_{0}\left(\Omega, \mathbb{E}^{3}\right) \times C_{0}(\Omega, \mathbb{R})$, 


$$
\begin{aligned}
& \widetilde{F}^{*}\left(\boldsymbol{\omega}^{0}, \boldsymbol{\omega}^{1}, \omega^{2}\right) \equiv \sup \left\{\int_{\Omega} \boldsymbol{\omega}^{0}: \mathbf{z} d x+\int_{\Omega} \boldsymbol{\omega}^{1}: \mathbf{p} d x\right. \\
& +\int_{\Omega} \omega^{2} t d x-\int_{\partial \Omega} \boldsymbol{\omega}^{0}:\left(\boldsymbol{\nu} \otimes \mathbf{u}_{\mid \partial \Omega}\right) d s+\int_{\Gamma_{1}} \mathbf{g} \cdot \mathbf{u} d s \\
& -\int_{\Gamma_{0}} I_{\{\boldsymbol{\nu} \otimes \widetilde{\mathbf{u}}=\mathbf{0}\}}(-\boldsymbol{\nu} \otimes \mathbf{u}) d s-\int_{\Gamma_{S}} h_{S}(x,-\boldsymbol{\nu} \otimes \mathbf{u}) d s \\
& -\int_{\Gamma_{W}} h_{W}(x,-\boldsymbol{\nu} \otimes \mathbf{u}) d s-\int_{\Omega} j(x, \mathbf{z}, \mathbf{p}, t) d x \\
& \left.(\mathbf{u}, \mathbf{z}, \mathbf{p}, t) \in C^{1}\left(\bar{\Omega}, \mathbb{R}^{3}\right) \times L^{1}\left(\Omega, \mathbb{E}^{3}\right) \times L^{1}\left(\Omega, \mathbb{E}^{3}\right) \times L^{1}(\Omega, \mathbb{R})\right\} \\
& =\sup \left\{-\int_{\partial \Omega} \boldsymbol{\omega}^{0}:\left(\boldsymbol{\nu} \otimes \mathbf{u}_{\mid \partial \Omega}\right) d s+\int_{\Gamma_{1}} \mathbf{g} \cdot \mathbf{u} d s\right. \\
& -\int_{\Gamma_{0}} I_{\{\boldsymbol{\nu} \otimes \widetilde{\mathbf{u}}=\mathbf{0}\}}(-\boldsymbol{\nu} \otimes \mathbf{u}) d s-\int_{\Gamma_{S}} h_{S}(x,-\boldsymbol{\nu} \otimes \mathbf{u}) d s \\
& \left.-\int_{\Gamma_{W}} h_{W}(x,-\boldsymbol{\nu} \otimes \mathbf{u}) d s \mid \mathbf{u} \in C^{1}\left(\bar{\Omega}, \mathbb{R}^{3}\right)\right\} \\
& +\sup \left\{\int_{\Omega} \boldsymbol{\omega}^{0}: \mathbf{z} d x+\int_{\Omega} \boldsymbol{\omega}^{1}: \mathbf{p} d x+\int_{\Omega} \omega^{2} t d x\right. \\
& \left.-\int_{\Omega} j(x, \mathbf{z}, \mathbf{p}, t) d x \mid(\mathbf{z}, \mathbf{p}, t) \in L^{1}\left(\Omega, \mathbb{E}^{3}\right) \times L^{1}\left(\Omega, \mathbb{E}^{3}\right) \times L^{1}(\Omega, \mathbb{R})\right\} \\
& =\int_{\Gamma_{1}} I_{\left\{\boldsymbol{\omega}^{0} \mid \boldsymbol{\omega}^{0} \cdot \boldsymbol{\nu}=\mathbf{g}\right\}}\left(\boldsymbol{\omega}^{0}\right) d s+\int_{\Gamma_{S}} h_{S}^{*}\left(x, \boldsymbol{\omega}^{0}\right) d s \\
& +\int_{\Gamma_{W}} h_{W}^{*}\left(x, \boldsymbol{\omega}^{0}\right) d s+\int_{\Omega} j^{*}\left(x, \boldsymbol{\omega}^{0}, \boldsymbol{\omega}^{1}, \omega^{2}\right) d x .
\end{aligned}
$$

We can apply [8, Theorem 1], since for $\mathbf{u}=\mathbf{0}$ we have $\int_{\Gamma_{1}} \mathbf{g} \cdot \mathbf{u} d s=0=$ $\int_{\Gamma_{0}} I_{\{\boldsymbol{\nu} \otimes \widetilde{\mathbf{u}}=\mathbf{0}\}}(-\boldsymbol{\nu} \otimes \mathbf{u}) d s, \int_{\Gamma_{S}} h_{S}(x,-\boldsymbol{\nu} \otimes \mathbf{u}) d s=0=\int_{\Gamma_{W}} h_{W}(x,-\boldsymbol{\nu} \otimes \mathbf{u}) d s$ (see (2.10) and (2.11)). Moreover, by (2.6) and $(2.7), \int_{\Omega} j^{*}(x, \mathbf{0}, \mathbf{0}, 0) d x$ is finite (cf. [22, Theorem 3A]).

There exists $\boldsymbol{\sigma}_{0} \in C\left(\bar{\Omega}, \mathbb{E}^{3}\right)$ such that $\boldsymbol{\sigma}_{0} \cdot \boldsymbol{\nu}=\mathbf{g}$ on $\Gamma_{1}, \boldsymbol{\sigma}_{0}=\mathbf{0}$ on $\Gamma_{W} \cup \Gamma_{S}$ and $\left(\boldsymbol{\sigma}_{0}(x), \mathbf{0}, 0\right) \in \mathcal{K}(x)$ for every $x \in \bar{\Omega}$ (see Assumption 3 ). Moreover, $\inf _{\left(\boldsymbol{\omega}^{0}, \boldsymbol{\omega}^{1}, \omega^{2}\right)} \widetilde{F}^{*}<\infty$, because inequality (2.5) holds.

Since the space $C\left(\bar{\Omega}, \mathbb{E}^{3}\right) \times C_{0}\left(\Omega, \mathbb{E}^{3}\right) \times C_{0}(\Omega, \mathbb{R})$ is PCU-stable, we obtain, basing upon the proof of $[8$, Theorem 1$]$, 


$$
\widetilde{F}^{* *}(\gamma(\mathbf{u}), \nabla \mathbf{u}, \widetilde{\boldsymbol{\mu}}, \mu)
$$

$$
\begin{aligned}
= & \sup \left\{\int_{\Omega}\left[\boldsymbol{\omega}^{0}:(\nabla \mathbf{u})_{a}+\boldsymbol{\omega}^{1}: \widetilde{\boldsymbol{\mu}}_{a}+\omega^{2} \mu_{a}-j^{*}\left(x, \boldsymbol{\omega}^{0}, \boldsymbol{\omega}^{1}, \omega^{2}\right)\right] d x\right. \\
& +\int_{\Omega}\left[\boldsymbol{\omega}^{0}:\left\{\frac{d(\nabla \mathbf{u})_{s}}{d\left|\left(\nabla \mathbf{u}_{s}, \widetilde{\boldsymbol{\mu}}_{s}, \mu_{s}\right)\right|}\right\}+\boldsymbol{\omega}^{1}:\left\{\frac{d \widetilde{\boldsymbol{\mu}}_{s}}{d\left|\left(\nabla \mathbf{u}_{s}, \widetilde{\boldsymbol{\mu}}_{s}, \mu_{s}\right)\right|}\right\}\right. \\
& \left.+\omega^{2}\left\{\frac{d \mu_{s}}{d\left|\left(\nabla \mathbf{u}_{s}, \widetilde{\boldsymbol{\mu}}_{s}, \mu_{s}\right)\right|}\right\}-I_{\mathcal{K}(x)}\left(\boldsymbol{\omega}^{0}, \boldsymbol{\omega}^{1}, \omega^{2}\right)\right] d\left|\left(\nabla \mathbf{u}_{s}, \widetilde{\boldsymbol{\mu}}_{s}, \mu_{s}\right)\right| \\
& -\int_{\Gamma_{1}} \boldsymbol{\omega}^{0}:(\boldsymbol{\nu} \otimes \gamma(\mathbf{u})) d s-\int_{\Gamma_{1}} I_{\left\{\boldsymbol{\omega}^{0} \mid \boldsymbol{\omega}^{0} \cdot \boldsymbol{\nu}=\mathbf{g}\right\}}\left(\boldsymbol{\omega}^{0}\right) d s \\
& +\int_{\Gamma_{S}}\left[-\boldsymbol{\omega}^{0}:(\boldsymbol{\nu} \otimes \gamma(\mathbf{u}))-h_{S}^{*}\left(\boldsymbol{\omega}^{0}\right)-I_{\mathcal{K}(x)}\left(\boldsymbol{\omega}^{0}, \boldsymbol{\omega}^{1}, \omega^{2}\right)\right] d s \\
& +\int_{\Gamma_{W}}\left[-\boldsymbol{\omega}^{0}:(\boldsymbol{\nu} \otimes \gamma(\mathbf{u}))-h_{W}^{*}\left(x, \boldsymbol{\omega}^{0}\right)-I_{\mathcal{K}(x)}\left(\boldsymbol{\omega}^{0}, \boldsymbol{\omega}^{1}, \omega^{2}\right)\right] d s \\
& +\int_{\Gamma_{0}}\left[-\boldsymbol{\omega}^{0}:(\boldsymbol{\nu} \otimes \gamma(\mathbf{u}))-I_{\mathcal{K}(x)}\left(\boldsymbol{\omega}^{0}, \boldsymbol{\omega}^{1}, \omega^{2}\right)\right] d s \mid \boldsymbol{\omega}^{0} \in C\left(\bar{\Omega}, \mathbb{E}^{3}\right), \\
= & \int_{\Omega} j\left(x,(\nabla \mathbf{u})_{a}, \widetilde{\boldsymbol{\mu}}_{a}, \mu_{a}\right) d x
\end{aligned}
$$$$
+\int_{\Omega} j_{\infty}\left(x, \frac{d(\nabla \mathbf{u})_{s}}{d\left|\left(\nabla \mathbf{u}_{s}, \widetilde{\boldsymbol{\mu}}_{s}, \mu_{s}\right)\right|}, \frac{d \widetilde{\boldsymbol{\mu}}_{s}}{d\left|\left(\nabla \mathbf{u}_{s}, \widetilde{\boldsymbol{\mu}}_{s}, \mu_{s}\right)\right|},\right.
$$

$$
\begin{array}{r}
\left.\frac{d \mu_{s}}{d\left|\left(\nabla \mathbf{u}_{s}, \widetilde{\boldsymbol{\mu}}_{s}, \mu_{s}\right)\right|}\right) d\left|\left(\nabla \mathbf{u}_{s}, \widetilde{\boldsymbol{\mu}}_{s}, \mu_{s}\right)\right| \\
-\int_{\Gamma_{1}} \mathbf{g} \cdot \boldsymbol{\gamma}(\mathbf{u}) d s+\int_{\Gamma_{S}}\left(h_{S}^{*}+I_{\mathcal{K}}\right)^{*}(x,-\boldsymbol{\nu} \otimes \boldsymbol{\gamma}(\mathbf{u}), \mathbf{0}, 0) d s \\
+\int_{\Gamma_{W}}\left(h_{W}^{*}+I_{\mathcal{K}}\right)^{*}(x,-\boldsymbol{\nu} \otimes \boldsymbol{\gamma}(\mathbf{u}), \mathbf{0}, 0) d s+\int_{\Gamma_{0}} j_{\infty}(x,-\boldsymbol{\nu} \otimes \boldsymbol{\gamma}(\mathbf{u}), \mathbf{0}, 0) d s
\end{array}
$$

for every $(\mathbf{u}, \widetilde{\boldsymbol{\mu}}, \mu) \in P_{q}^{3} B V(\Omega)$ and every $q>0$. Finally, $\widetilde{F}^{* *}$ is l.s.c. in the topology (3.1)-(3.2) [and in the topology (3.11)-(3.12)], because $\widetilde{F}^{* *}$ is a supremum of affine functionals continuous in the topology (3.1)-(3.2) (cf. Corollary 6).

Proof of Theorem 3. Because the functional $\widetilde{F}^{* *}$ is coercive, there exist $q_{0}>0$ and a bounded sequence $\left\{\left(\mathbf{u}_{m}, \widetilde{\boldsymbol{\mu}}_{m}, \mu_{m}\right)\right\}_{m \in \mathbb{N}} \subset P_{q_{0}}^{3} B V(\Omega)$ which minimize $\widetilde{F}^{* *}$. By Theorem 7 and metrizability of $P_{q_{0}}^{3} B V(\Omega)$ (cf. proof of Theorem 7) there exist a subsequence $\left\{\left(\mathbf{u}_{m_{p}}, \widetilde{\boldsymbol{\mu}}_{m_{p}}, \mu_{m_{p}}\right)\right\}_{p \in \mathbb{N}} \subset P_{q_{0}}^{3} B V(\Omega)$ 
and $\left(\mathbf{u}_{0}, \widetilde{\boldsymbol{\mu}}_{0}, \mu_{0}\right) \in P_{q_{0}}^{3} B V(\Omega)$ such that $\left\{\left(\mathbf{u}_{m_{p}}, \widetilde{\boldsymbol{\mu}}_{m_{p}}, \mu_{m_{p}}\right)\right\}_{p \in \mathbb{N}}$ converges to $\left(\mathbf{u}_{0}, \widetilde{\boldsymbol{\mu}}_{0}, \mu_{0}\right)$ in the topology (3.1)-(3.2). In view of (2.9), (3.1), (3.2) and (2.16) the functional $\widetilde{F}^{* *}$ is l.s.c. in the topology (3.1)-(3.2) and we obtain $(2.32)$.

We specify the vector $\mathbf{u}$ in (2.24) as the displacement field.

EXAmple 1. Let $j(x, \mathbf{z}, \mathbf{p}, t)=j_{2}(x, \mathbf{z}, \mathbf{p})+\alpha t^{2}$ for every $(\mathbf{z}, \mathbf{p}, t) \in \mathbb{E}^{3} \times$ $\mathbb{E}^{3} \times \mathbb{R}$ and for $d x$-a.e. $x \in \Omega$, where $\alpha>0$ and $j_{2}$ is a convex, nonnegative normal integrand. Let $\mathcal{K}_{2}(x) \equiv\left\{\left(\mathbf{z}^{*}, \mathbf{p}^{*}\right) \in \mathbb{E}^{3} \times \mathbb{E}^{3} \mid j_{2}^{*}\left(x, \mathbf{z}^{*}, \mathbf{p}^{*}\right)<\infty\right\}$ for $d x$-a.e. $x \in \Omega$, where

$$
j_{2}^{*}\left(x, \mathbf{z}^{*}, \mathbf{p}^{*}\right)=\sup \left\{\mathbf{z}: \mathbf{z}^{*}+\mathbf{p}: \mathbf{p}^{*}-j_{2}(x, \mathbf{z}, \mathbf{p}) \mid(\mathbf{z}, \mathbf{p}) \in \mathbb{E}^{3} \times \mathbb{E}^{3}\right\}
$$

for every $\left(\mathbf{z}^{*}, \mathbf{p}^{*}\right) \in \mathbb{E}^{3} \times \mathbb{E}^{3}$ and for $d x$-a.e. $x \in \Omega$. Then

$$
\begin{array}{r}
\left(h_{S}^{*}+I_{\mathcal{K}_{2}}\right)^{*}(x,-\boldsymbol{\nu} \otimes \gamma(\mathbf{u}), \mathbf{0})=\sup \left\{-\mathbf{z}^{*}:(\boldsymbol{\nu} \otimes \gamma(\mathbf{u}))-h_{S}^{*}\left(\mathbf{z}^{*}\right) \mid\right. \\
\left.\left(\mathbf{z}^{*}, \mathbf{0}\right) \in \mathcal{K}_{2}(x) \subset \mathbb{E}^{3} \times \mathbb{E}^{3}\right\}
\end{array}
$$

and

$$
\begin{aligned}
\left(h_{W}^{*}+I_{\mathcal{K}_{2}}\right)^{*}(x,-\boldsymbol{\nu} \otimes \boldsymbol{\gamma}(\mathbf{u}), \mathbf{0})=\sup \left\{-\mathbf{z}^{*}:(\boldsymbol{\nu} \otimes \boldsymbol{\gamma}(\mathbf{u}))-h_{W}^{*}\left(x, \mathbf{z}^{*}\right) \mid\right. \\
\left.\left(\mathbf{z}^{*}, \mathbf{0}\right) \in \mathcal{K}_{2}(x) \subset \mathbb{E}^{3} \times \mathbb{E}^{3}\right\},
\end{aligned}
$$

where $h_{S}^{*}$ and $h_{W}^{*}$ are given by (2.22) and (2.23). We define

$$
\left(j_{2}\right)_{\infty}(x, \mathbf{z}, \mathbf{p}) \equiv \sup \left\{\mathbf{z}: \mathbf{z}^{*}+\mathbf{p}: \mathbf{p}^{*}-I_{\mathcal{K}_{2}(x)}\left(\mathbf{z}^{*}, \mathbf{p}^{*}\right) \mid \mathbf{z}^{*} \in \mathbb{E}^{3}, \mathbf{p}^{*} \in \mathbb{E}^{3}\right\}
$$

for $(\mathbf{z}, \mathbf{p}) \in \mathbb{E}^{3} \times \mathbb{E}^{3}$ and $x \in \Omega$. Therefore for every $(\mathbf{u}, \widetilde{\boldsymbol{\mu}}, \mu) \in P_{q}^{3} B V(\Omega)$,

$$
\begin{aligned}
\widetilde{F}^{* *}(\nabla \mathbf{u}, \widetilde{\boldsymbol{\mu}}, \mu)= & -\int_{\Gamma_{1}} \mathbf{g} \cdot \boldsymbol{\gamma}(\mathbf{u}) d s+\int_{\Gamma_{0}}\left(j_{2}\right)_{\infty}(x,-\boldsymbol{\nu} \otimes \gamma(\mathbf{u}), \mathbf{0}) d s \\
& +\int_{\Gamma_{S}}\left(h_{S}^{*}+I_{\mathcal{K}_{2}}\right)^{*}(x,-\boldsymbol{\nu} \otimes \gamma(\mathbf{u}), \mathbf{0}) d s+\alpha \int_{\Omega} \mu^{2} d x \\
& +\int_{\Gamma_{W}}\left(h_{W}^{*}+I_{\mathcal{K}_{2}}\right)^{*}(x,-\boldsymbol{\nu} \otimes \gamma(\mathbf{u}), \mathbf{0}) d s \\
& +\int_{\Omega} j_{2}\left(x,(\nabla \mathbf{u})_{a}, \widetilde{\boldsymbol{\mu}}_{a}\right) d x \\
& +\int_{\Omega}\left(j_{2}\right)_{\infty}\left(x, \frac{d(\nabla \mathbf{u})_{s}}{d\left|\left(\nabla \mathbf{u}_{s}, \widetilde{\boldsymbol{\mu}}_{s}\right)\right|}, \frac{d \widetilde{\boldsymbol{\mu}}_{s}}{d\left|\left(\nabla \mathbf{u}_{s}, \widetilde{\boldsymbol{\mu}}_{s}\right)\right|}\right) d\left|\left(\nabla \mathbf{u}_{s}, \widetilde{\boldsymbol{\mu}}_{s}\right)\right|
\end{aligned}
$$

if $\mu$ is absolutely continuous with respect to $d x$ and $\mu \in L^{2}(\Omega, \mathbb{R})$, and $\widetilde{F}^{* *}(\nabla \mathbf{u}, \tilde{\boldsymbol{\mu}}, \mu)=\infty$ otherwise.

In Example 1 we have dealt with the body whose elastic-plastic potential is finite even in the case when the volume of the body after compression 
could be reduced to zero. Now (Example 2) we consider a more realistic case, in which the potential in the case of volume reduced to zero becomes infinite.

Example 2. We assume that the body is clamped on $\Gamma_{0}$ (i.e. $\mathbf{u}=\mathbf{0}$ on $\Gamma_{0}$ ), and on $\Gamma_{1}$ the boundary force $\mathbf{g}$ is prescribed.

Moreover, let the multifunction $\mathcal{K}$ satisfy (2.27). Then (2.29) and (2.31) hold, and the elastic-plastic potential is given by

$$
j(x, \mathbf{z}, \mathbf{p}, t)= \begin{cases}j_{3}(x, \mathbf{z}, \mathbf{p})+\alpha t^{2} & \text { if } t \geq 0 \\ j_{3}(x, \mathbf{z}, \mathbf{p})-\alpha_{1} \frac{t}{t+1} & \text { if }-1<t<0 \\ \infty & \text { if } t \leq-1\end{cases}
$$

where $\alpha, \alpha_{1}>0$ and $j_{3}$ is a convex, nonnegative normal integrand such that

$$
\begin{aligned}
0<\alpha_{2} & <\liminf _{|\mathbf{z}| \rightarrow \infty} j_{3}(x, \mathbf{z}, \mathbf{p}) /|\mathbf{z}| \\
& \leq \limsup _{|\mathbf{z}| \rightarrow \infty} j_{3}(x, \mathbf{z}, \mathbf{p}) /|\mathbf{z}|<\infty
\end{aligned}
$$

for $\alpha_{2} \in \mathbb{R}$ and every (fixed) $x \in \Omega, \mathbf{p} \in \mathbb{E}^{3}$.

EXAMPLE 3. Let

$$
j_{4}^{*}\left(x, \mathbf{z}^{*}, \mathbf{p}^{*}\right)= \begin{cases}\theta(x)\left(\left\|\mathbf{z}^{*}\right\|_{\mathbb{E}^{3}}^{2}+\left\|\mathbf{p}^{*}\right\|_{\mathbb{E}^{3}}^{2}\right) & \text { if } \mathbf{z}^{*} \in B_{\mathbb{E}^{3}}(0,1) \\ & \text { and } \mathbf{p}^{*} \in B_{\mathbb{E}^{3}}(0,1), \\ \infty & \text { otherwise, }\end{cases}
$$

where $\theta: \Omega \rightarrow \mathbb{R}$ is a positive Borel function. Then $j_{4}(x, \mathbf{z}, \mathbf{p})=j_{4}^{* *}(x, \mathbf{z}, \mathbf{p})=$ $\sup \left\{\mathbf{z}: \mathbf{z}^{*}+\mathbf{p}: \mathbf{p}^{*}-j_{4}^{*}\left(x, \mathbf{z}^{*}, \mathbf{p}^{*}\right) \mid\left\|\mathbf{z}^{*}\right\|_{\mathbb{E}^{3}} \leq 1,\left\|\mathbf{p}^{*}\right\|_{\mathbb{E}^{3}} \leq 1\right\}$. The functional $j_{4}$ has a linear growth at infinity, but it is not a positive monotonic function. Define

$$
j(x, \mathbf{z}, \mathbf{p}, t) \equiv \begin{cases}j_{4}(x, \mathbf{z}, \mathbf{p})+\alpha t^{2} & \text { if } t \geq 0 \\ j_{4}(x, \mathbf{z}, \mathbf{p})-\alpha_{1} \frac{t}{t+1} & \text { if }-1<t<0 \\ \infty & \text { if } t \leq-1\end{cases}
$$

where $\alpha, \alpha_{1}>0$. The last functional describes a real elastic-plastic material in the range of finite displacements. Moreover, in (3.23) we can replace $j_{4}$ by

$$
j_{5}^{*}\left(x, \mathbf{z}^{*}, \mathbf{p}^{*}\right)= \begin{cases}\theta(x)\left(\left\|\mathbf{z}^{*}\right\|_{\mathbb{E}^{3}}^{2}+\left\|\mathbf{p}^{*}\right\|_{\mathbb{E}^{3}}^{2}\right) & \text { if }\left(\mathbf{z}^{*}\right)^{D} \in B_{\mathbb{E}^{3}}(0,1) \\ & \text { and } \mathbf{p}^{*} \in B_{\mathbb{E}^{3}}(0,1), \\ \infty & \text { otherwise }\end{cases}
$$

where $\left(\mathbf{z}^{*}\right)^{D}$ is the deviator part of $\mathbf{z}^{*}$ and $j_{5}=j_{5}^{* *}$. 
[1] L. Ambrosio and G. Dal Maso, On the relaxation in $B V\left(\Omega, R^{m}\right)$ of quasi-convex integrals, J. Funct. Anal. 109 (1992), 76-97.

[2] L. Ambrosio and D. Pallara, Integral representations of relaxed functionals on $B V\left(R^{n}, R^{k}\right)$ and polyhedral approximation, Indiana Univ. Math. J. 42 (1993), 295321.

[3] J. M. Ball, Convexity conditions and existence theorems in nonlinear elasticity, Arch. Rat. Mech. Anal. 63 (1977), 337-403.

[4] J. L. Bojarski, The relaxation of Signorini problems in Hencky plasticity, I: threedimensional solid, Nonlinear Anal. 29 (1997), 1091-1116.

[5] J. L. Bojarski and H. De Schepper, Polyconvex functionals with linear growth at infinity, Int. J. Pure Appl. Math. 8 (2003), 55-74.

[6] G. Bouchitté, I. Fonseca and L. Mascarenhas, A global method for relaxation, Arch. Rat. Mech. Anal. 145 (1998), 51-98.

[7] - - - - , Relaxation of variational problems under trace constraints, Nonlinear Anal. 49 (2002), 221-246.

[8] G. Bouchitté and M. Valadier, Integral representation of convex functionals on a space of measures, J. Funct. Anal. 80 (1988), 398-420.

[9] G. Dal Maso and C. Sbordone, Weak lower semicontinuity of polyconvex integrals: a borderline case, Math. Z. 218 (1995) 603-609.

[10] N. Dunford and J. T. Schwartz, Linear Operators, Part I, Interscience Publ., New York, 1958.

[11] G. Duvaut et J.-L. Lions, Les Inéquations en Mécanique et en Physique, Dunod, Paris, 1972.

[12] I. Ekeland and R. Temam, Convex Analysis and Variational Problems, NorthHolland, Amsterdam and New York, 1976.

[13] R. Engelking, General Topology, PWN-Polish Sci. Publ., Warszawa, 1977.

[14] I. Fonseca and S. Müller, Relaxation of quasiconvex functionals in $B V\left(\Omega, R^{p}\right)$ for integrands $f(x, u, \nabla u)$, Arch. Rat. Mech. Anal. 123 (1993), 1-49.

[15] M. Giaquinta, G. Modica and J. Souček, Cartesian currents, weak diffeomorphisms and existence theorems in nonlinear elasticity, Arch. Rat. Mech. Anal. 106 (1989), 97-159.

[16] —, - - -, Erratum and addendum to [15], ibid. 109 (1990), 385-392.

[17] - , - - Cartesian Currents in the Calculus of Variations, Vol. II: Variational Integrals, Ergeb. Math. Grenzgeb. 38, Springer, Berlin, 1998.

[18] E. Giusti, Minimal Surfaces and Functions of Bounded Variation, lecture notes written by G. H. Williams, Dept. Math., Australian National Univ., Canberra, 1977.

[19] T. Iwaniec and A. Lutoborski, Polyconvex functionals for nearly conformal deformations, SIAM J. Math. Anal. 27 (1996), 609-619.

[20] S. Müller, A remark on the distributional determinant, C. R. Acad. Sci. Paris Sér. I Math. 311 (1990), 13-17.

[21] W. Nowacki, Mechanics of Structures, Vol. 3, PWN-Polish Sci. Publ., Warszawa, 1975 (in Polish).

[22] R. T. Rockafellar, Integral functionals, normal integrands and measurable selections, in: Nonlinear Operators and the Calculus of Variations, Lecture Notes in Math. 543, Springer, Berlin, 1975, 157-207.

[23] R. Temam, Mathematical Problems in Plasticity, Gauthier-Villars, Paris, 1985. 
[24] R. Temam and G. Strang, Functions of bounded deformation, Arch. Rat. Mech. Anal. 75 (1980), 7-21.

Department of Applied Mathematics

Warsaw Agricultural University-SGGW

Nowoursynowska 159

02-787 Warszawa, Poland

E-mail: JarekLBojarski@poczta.onet.pl

Received on 23.8.2005;

revised version on 25.11.2005 